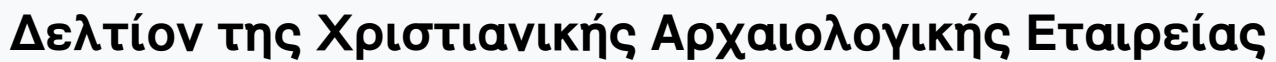

Tó 30 (2009)

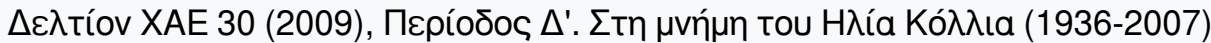

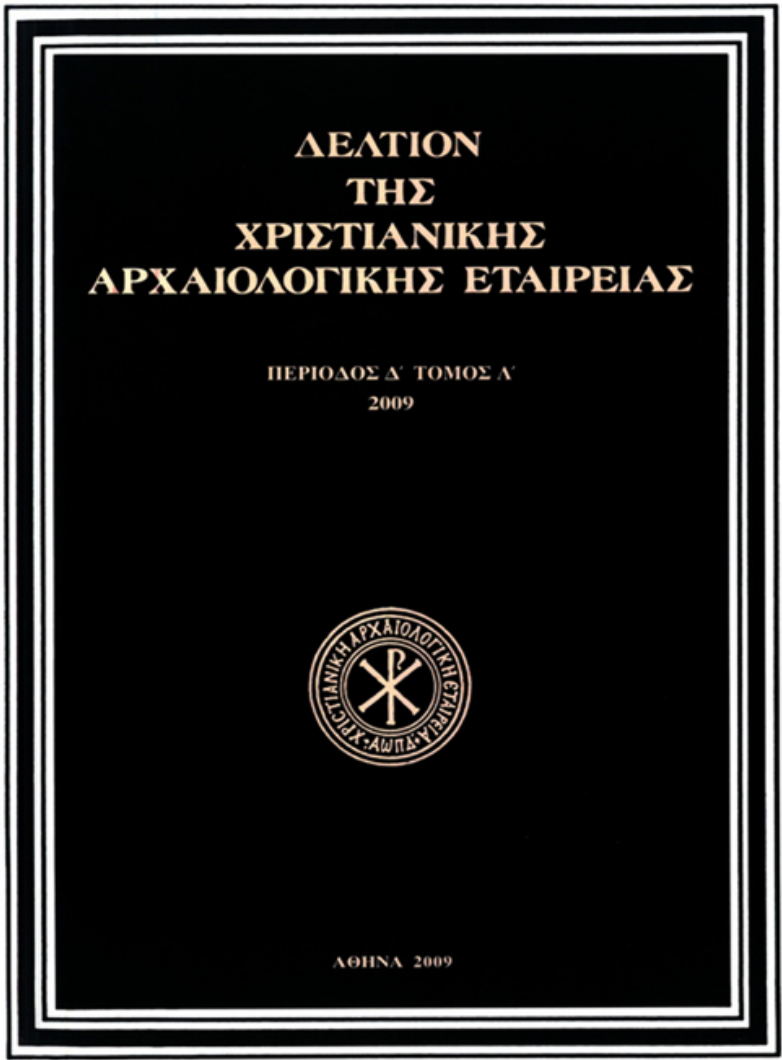

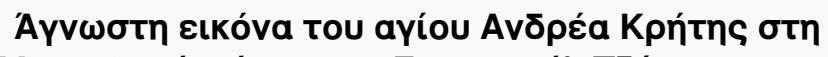

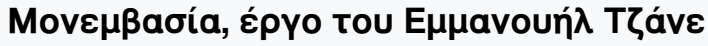

Mapía АГРЕВH

doi: $10.12681 /$ dchae. 656

\section{Bıß入ıорафıкń avaфора́:}

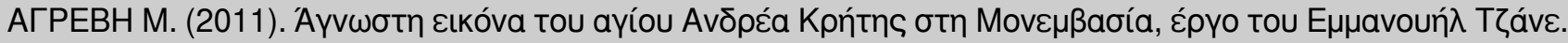

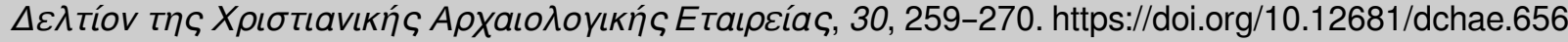




\section{$\triangle \mathrm{E} \Lambda \mathrm{TION}$ TH $\Sigma$ XPI $\Sigma$ TIANIKH $\Sigma$ APXAIO $\Lambda$ OГIKH $\Sigma$ ETAIPEIA $\Sigma$}

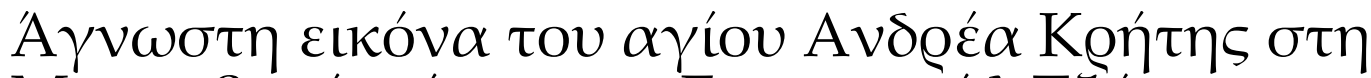

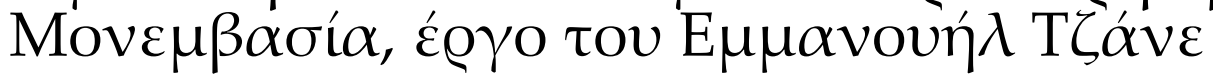

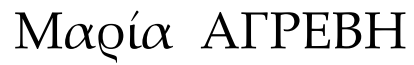

Пeqíodos $\Delta^{\prime}$, Tómos $\Lambda^{\prime}(2009) \bullet \Sigma \varepsilon \lambda$. 259-270 A@HNA 2009 


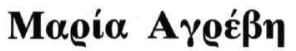

\section{AГN $\Omega \Sigma$ TH EIKONA TOY AГIOY AN $\triangle$ PEA KPHTH $\Sigma$ $\Sigma$ TH MONEMBA $\Sigma$ IA, EPГO TOY EMMANOYHA TZANE*}

$\mathrm{H}$

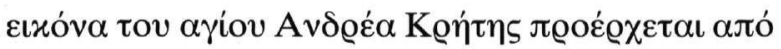

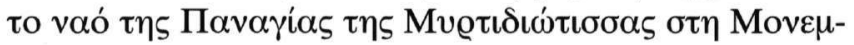

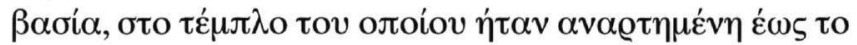

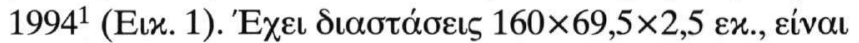

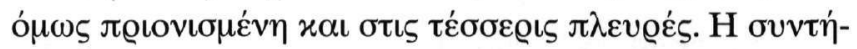

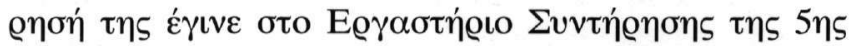

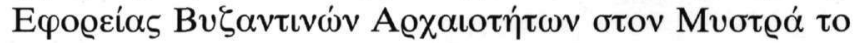

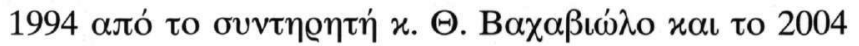

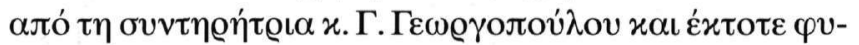

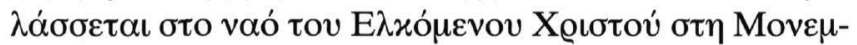
$\beta \alpha \sigma i \alpha$.

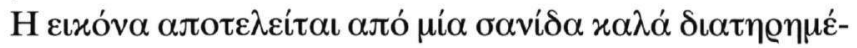

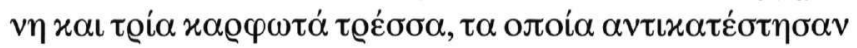

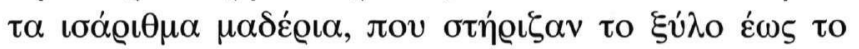

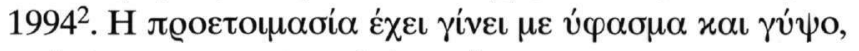

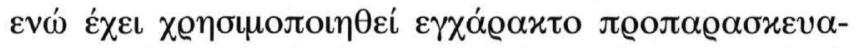

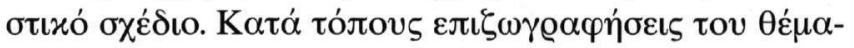

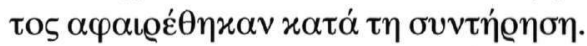

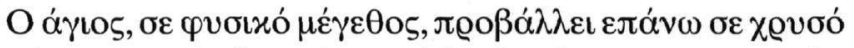

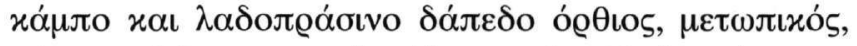

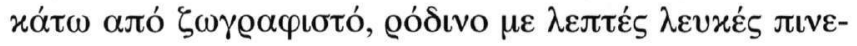

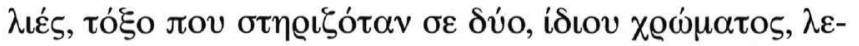

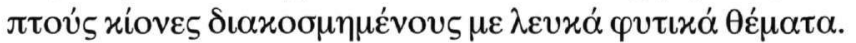

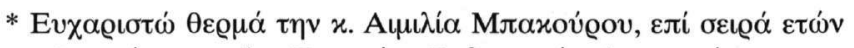

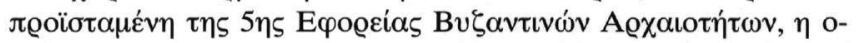

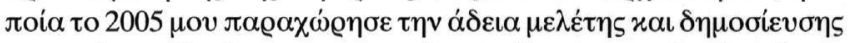

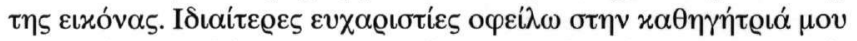

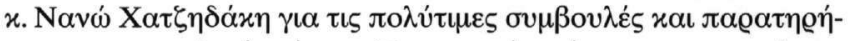

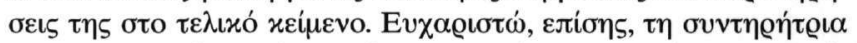

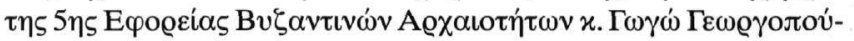

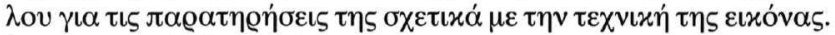

${ }^{1} \Gamma$ la to voó $\beta \lambda$. xv@íws R. Tranquair, «Laconia, the Fortresses:

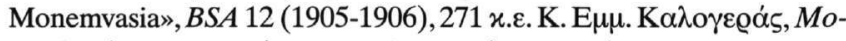

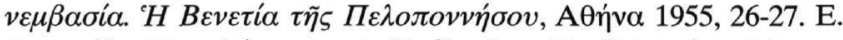

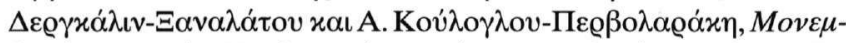

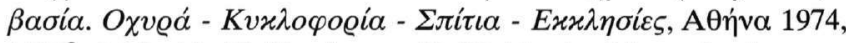

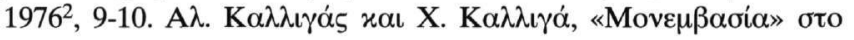

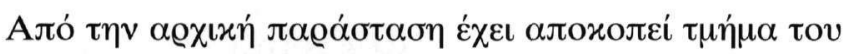

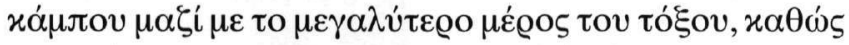

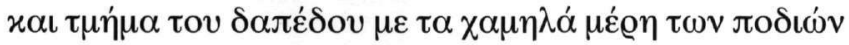

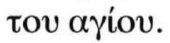

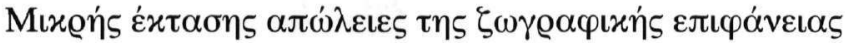

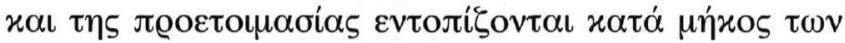

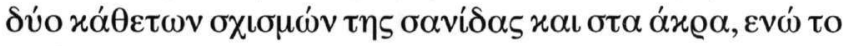

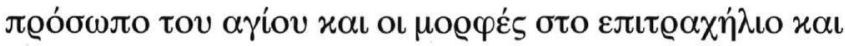

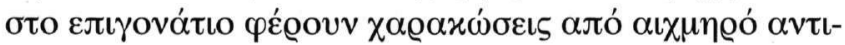

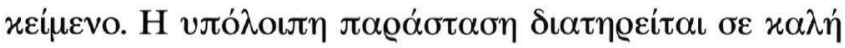

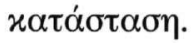

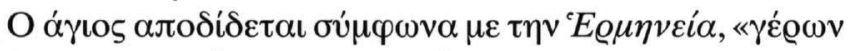

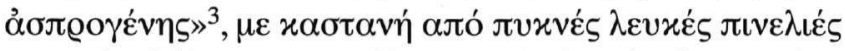

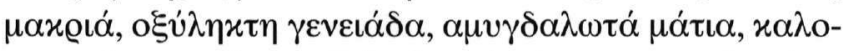

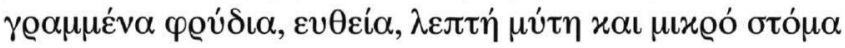

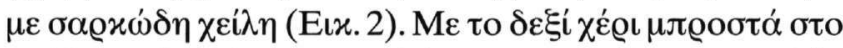

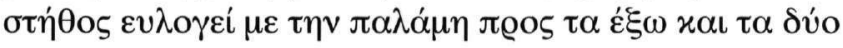

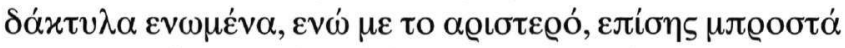

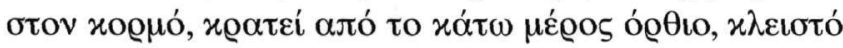

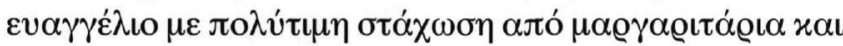

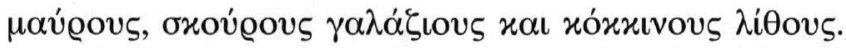

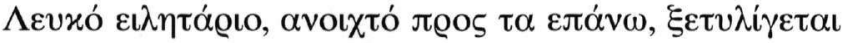

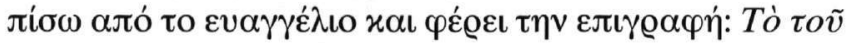

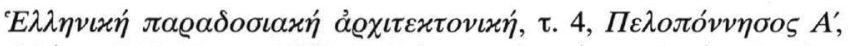

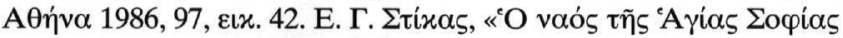

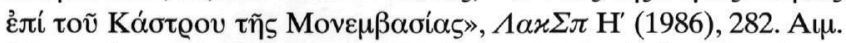

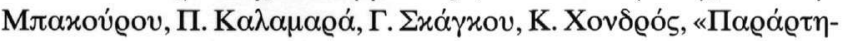

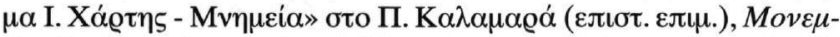

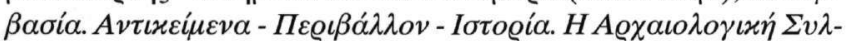

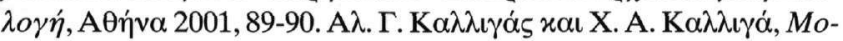

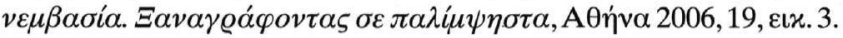

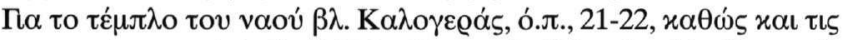

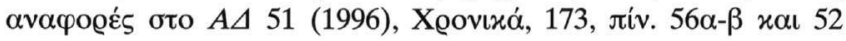
(1997), Xอovıxá, 221.

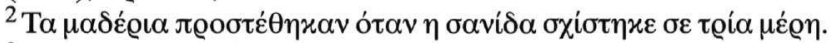

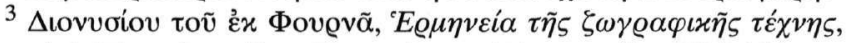

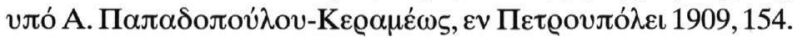


MAPIA АГРЕВН

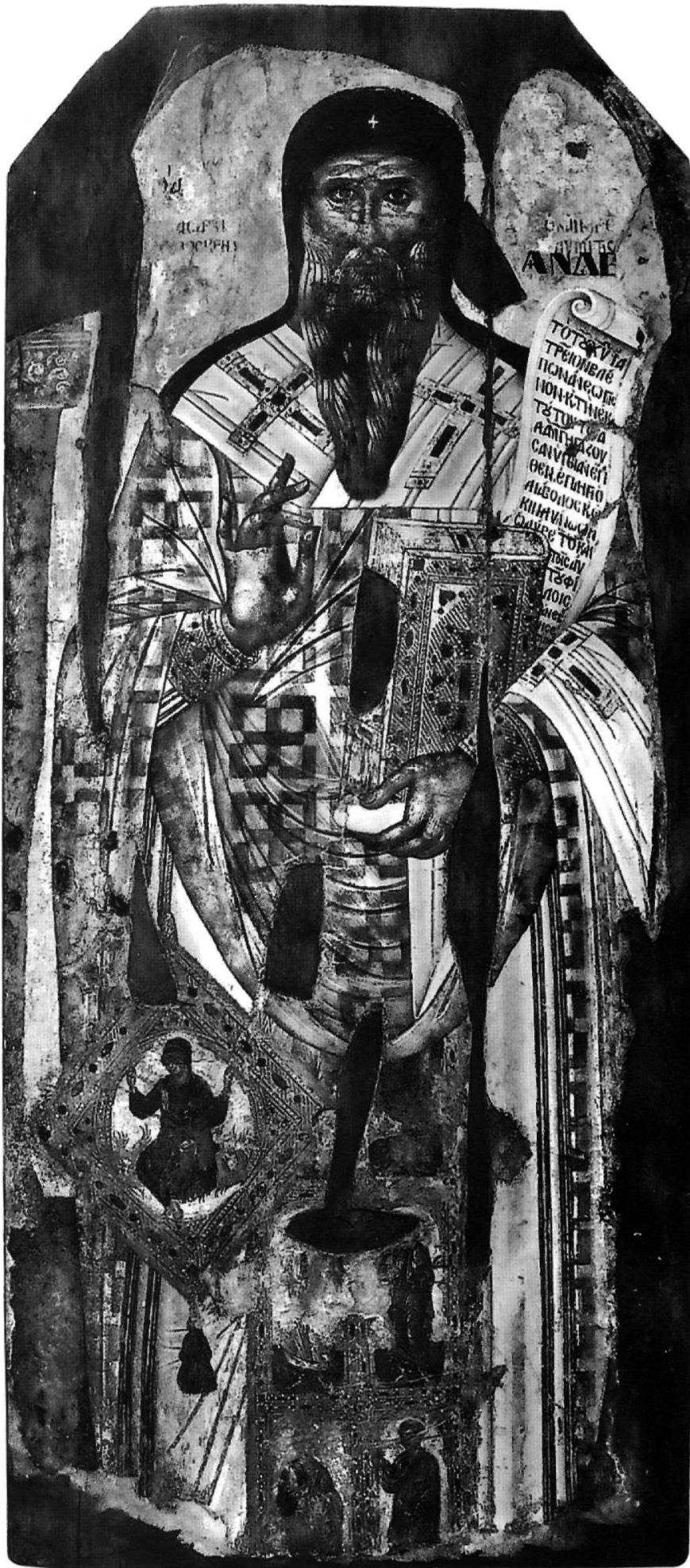

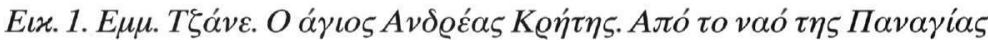

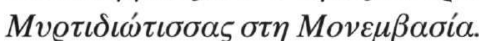




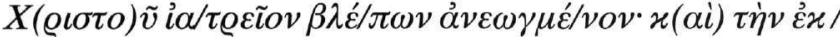

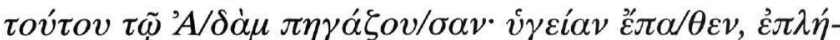

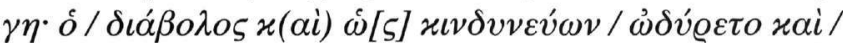

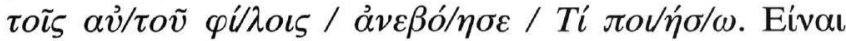

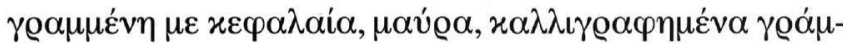

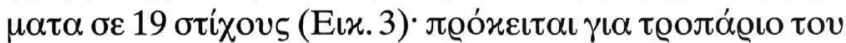

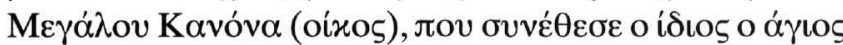

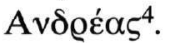

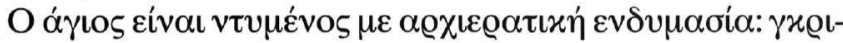

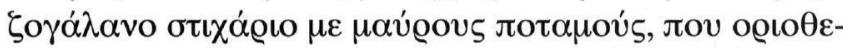

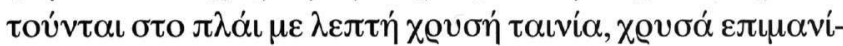

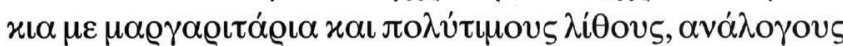

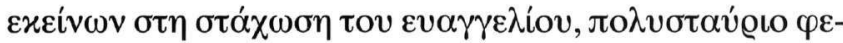

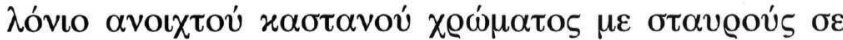

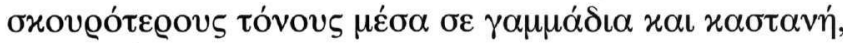

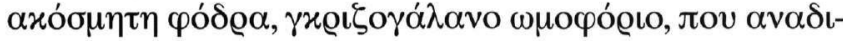

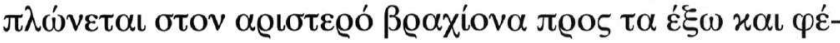

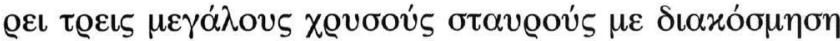

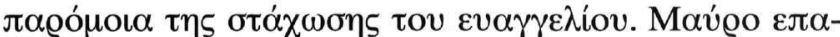

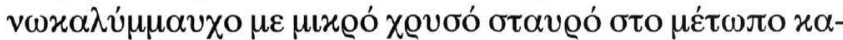

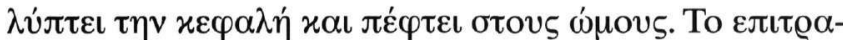

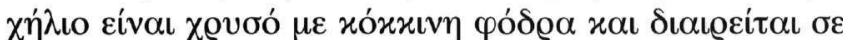

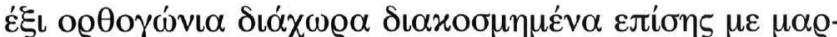

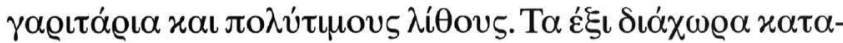

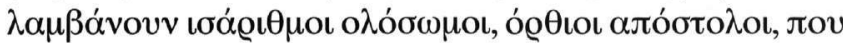

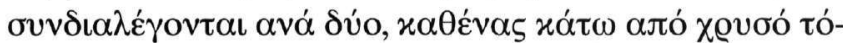

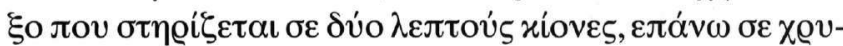

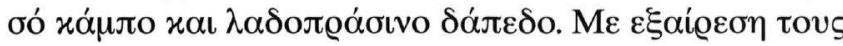

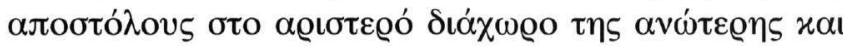

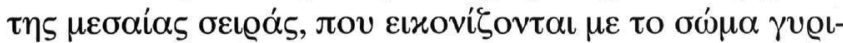

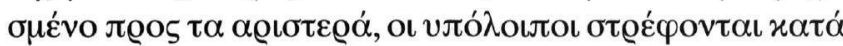

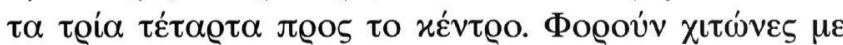

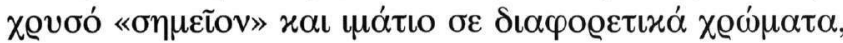

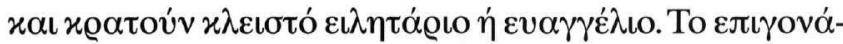

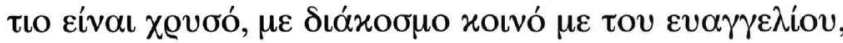

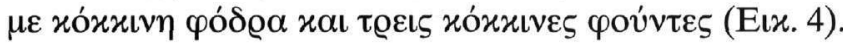

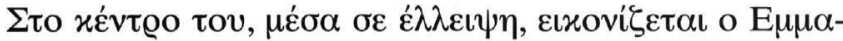

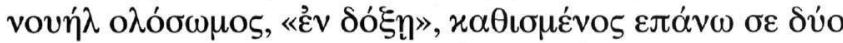

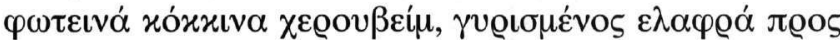

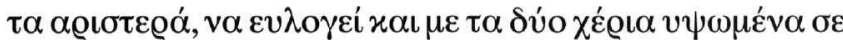

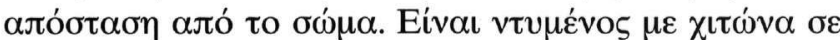

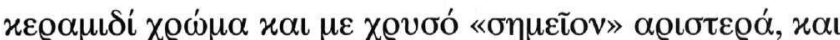

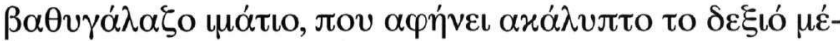

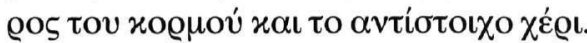

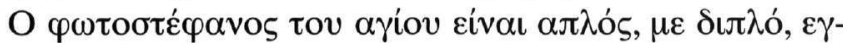

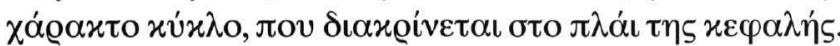

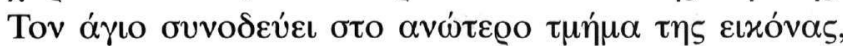

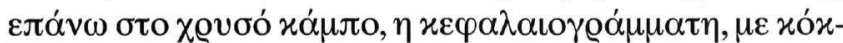

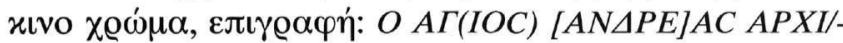

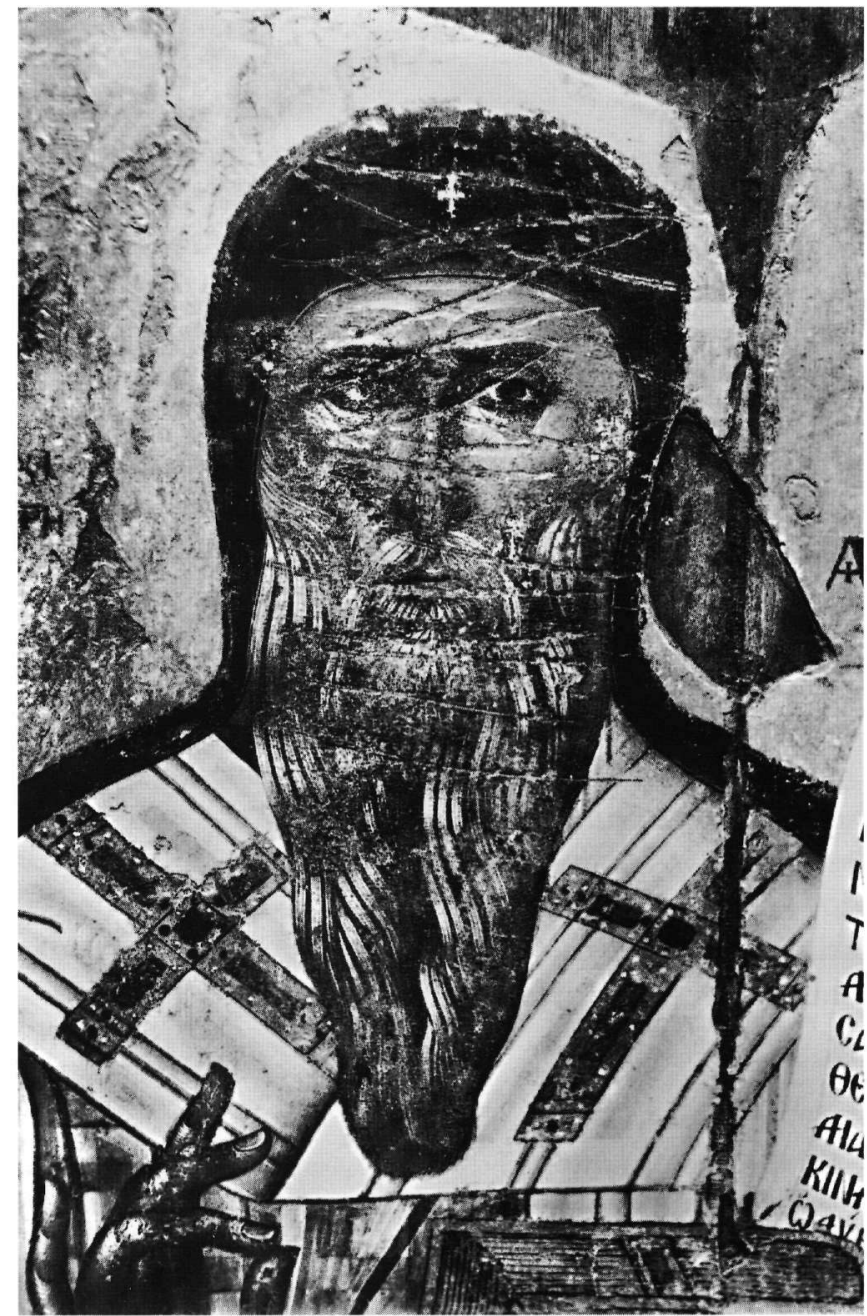

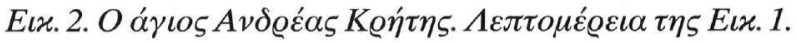

[ЕПICКОП]ОС КРНТ]НС]/O КАIIЕPO/[CO]АYMITHC.

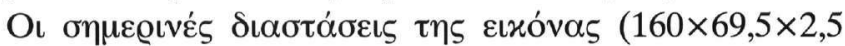

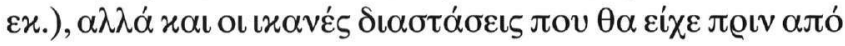

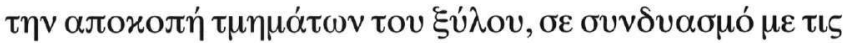

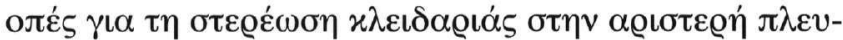

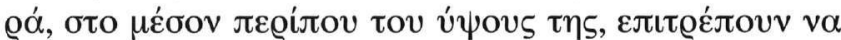

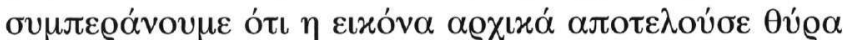
$\pi \alpha \varrho \alpha \beta \eta \dot{\eta} \mu \alpha \tau o \zeta \alpha ́ \gamma v \omega \sigma \tau o v \tau \varepsilon \dot{\mu} \mu \pi \lambda \circ v$.

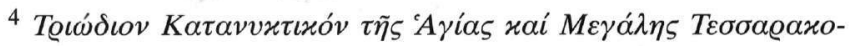

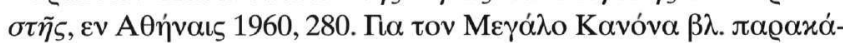

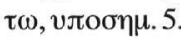




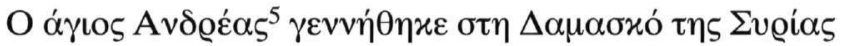

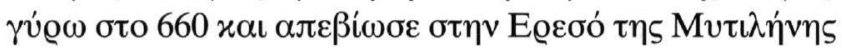

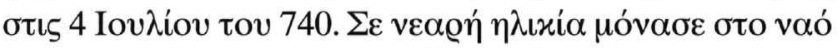

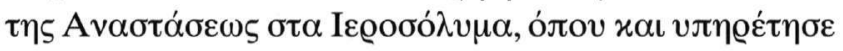

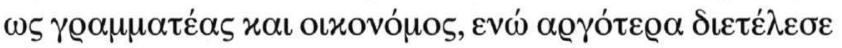

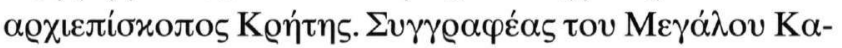

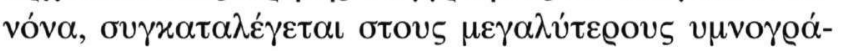

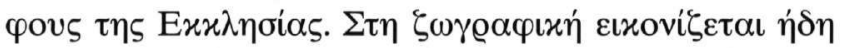

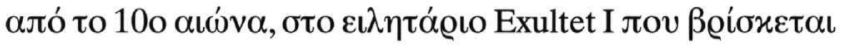

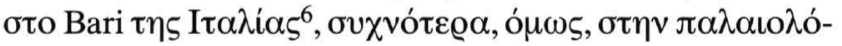

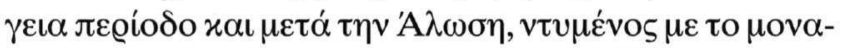

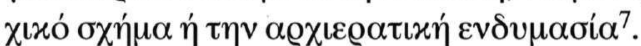

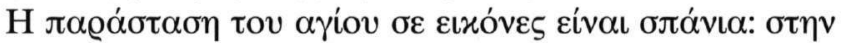

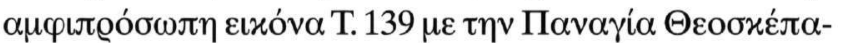

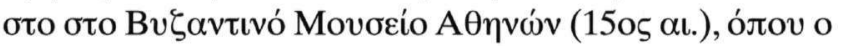

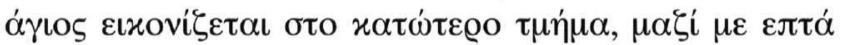

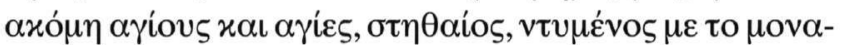

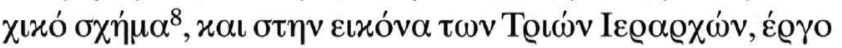

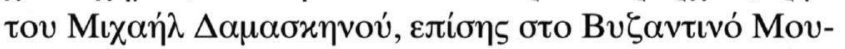

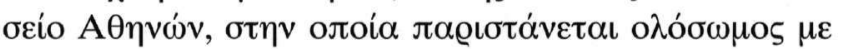

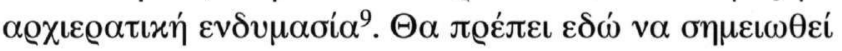

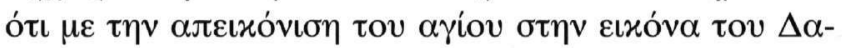

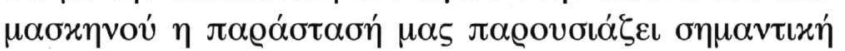

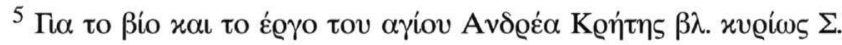

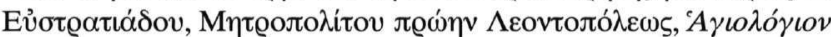

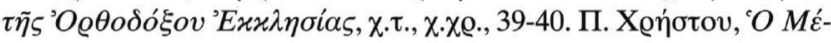

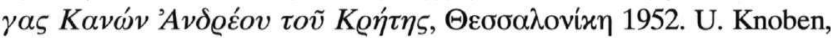

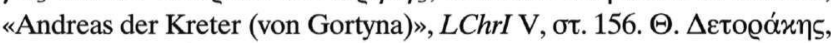

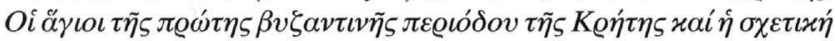

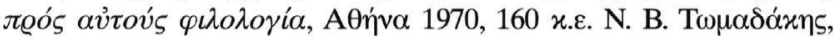

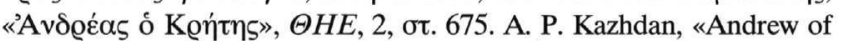

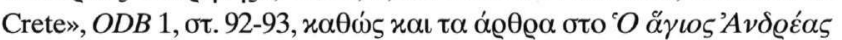

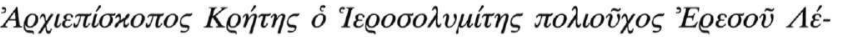

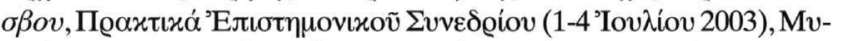

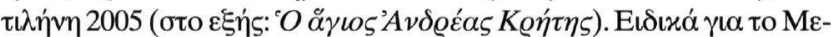

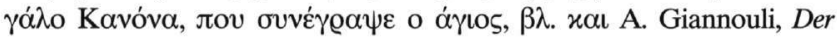
Kommentar des Akkakios Sabaïtes zum Großen Kanon des Andreas von Kreta. Studien zur Überlieferung und Quellen. Text auf der Basis des Codex

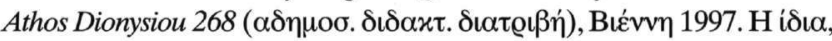
«Die Kommentartradition zum Grossen Kanon des Andreas von Kreta:

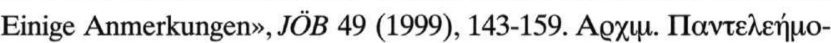

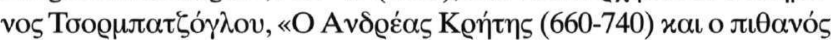

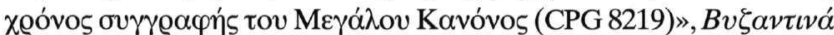

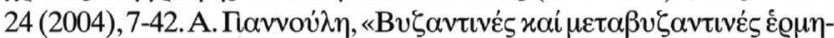

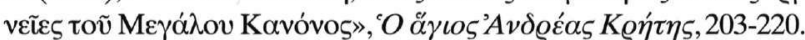

${ }^{6}$ M. Avery, The Exultet Rolls of South Italy, Princeton 1936, II, Jiv. IX.

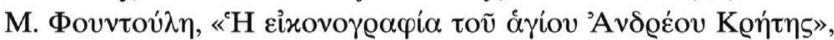

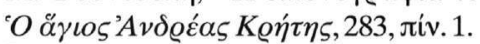

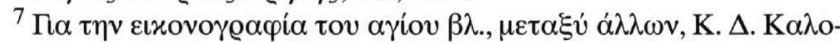

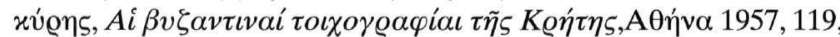

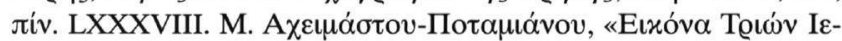

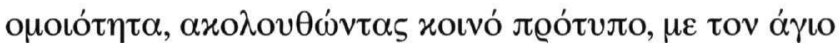

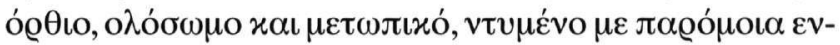

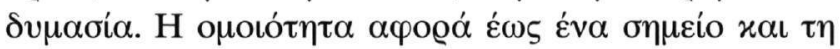

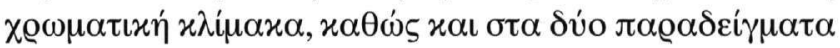

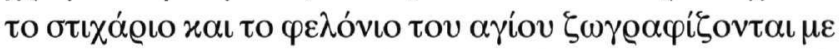

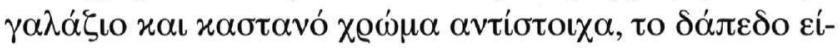

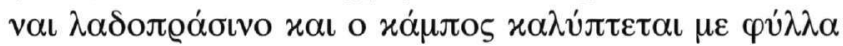

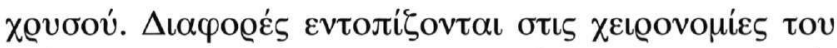

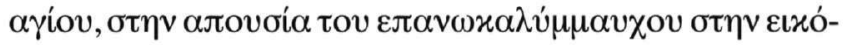

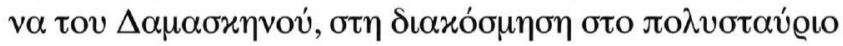

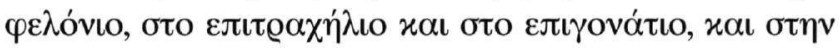

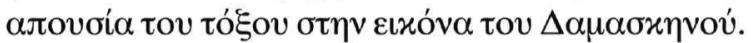

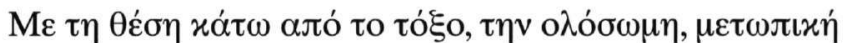

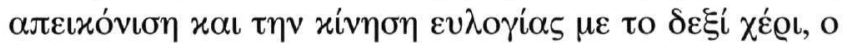

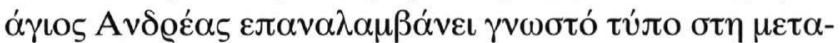

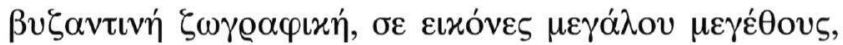

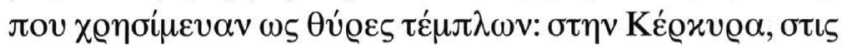

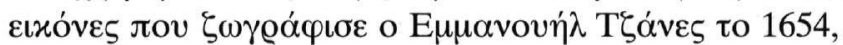

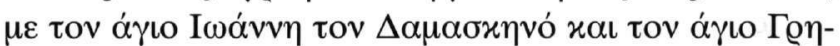

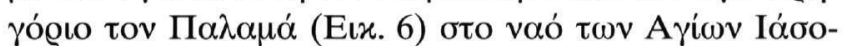

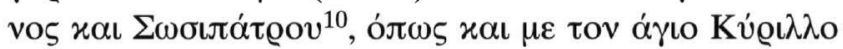

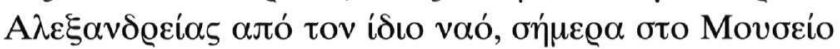

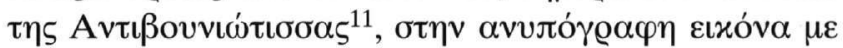

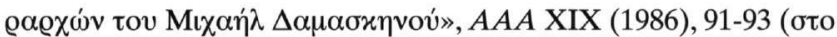

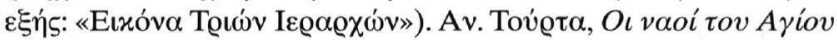

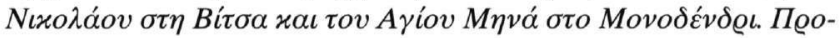

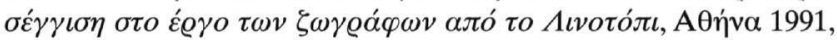
63. A. Semoglou, Le décor mural de la chapelle athonite de Saint Nicolas (1560). Application d'un nouveau langage pictural par le peintre thébain

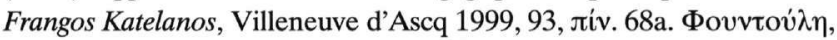

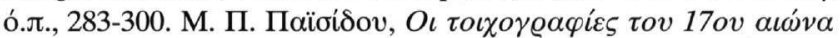

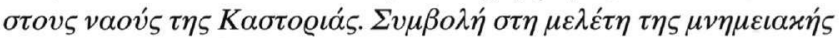

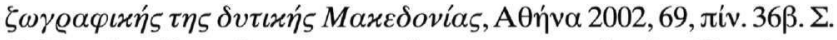

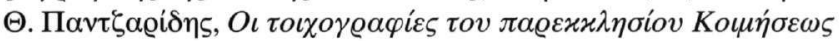

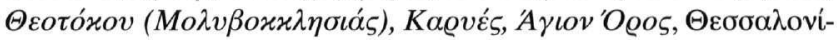
xү 2006, 64-65, عเx. 45.

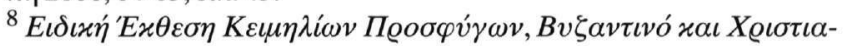

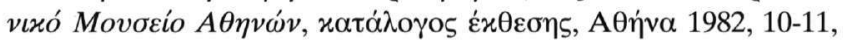

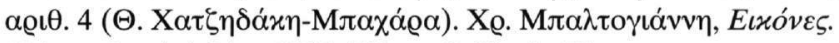

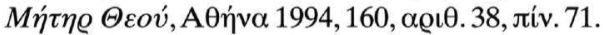

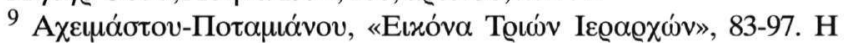

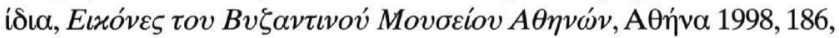
$\alpha \varrho \iota$. 56. Riflessi di Bisanzio. Capolavori d'arte dal XV al XVIII secolo dal Museo Bizantino e Cristiano di Atene, Musei Capitolini, Palazzo Caffarelli,

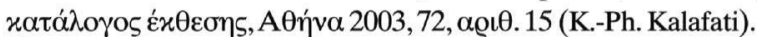

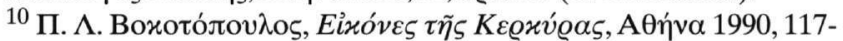

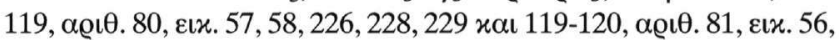

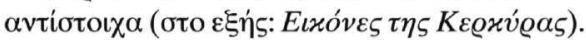

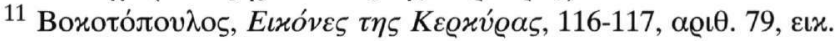

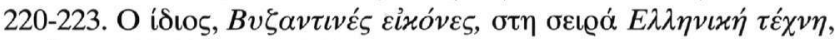
A $\theta \dot{v} v \alpha$ 1995, 228, $\alpha \varrho \iota \theta .165$. 


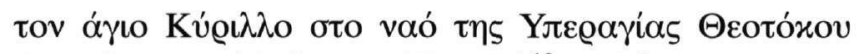

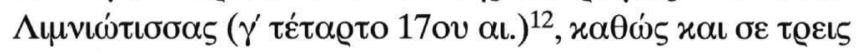

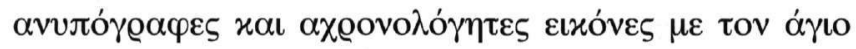

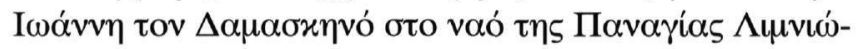

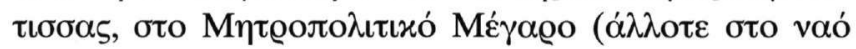

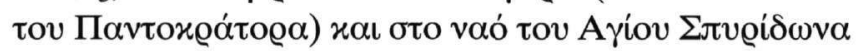

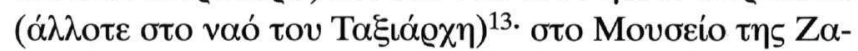

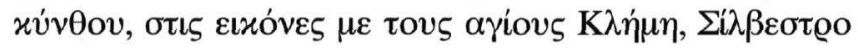

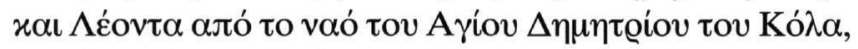

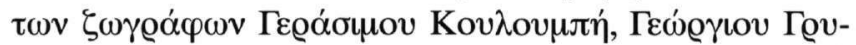

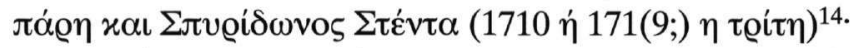

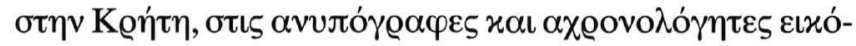

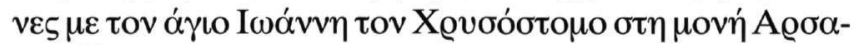

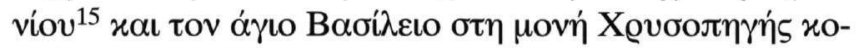

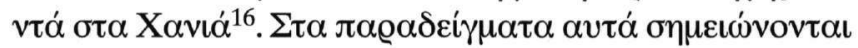

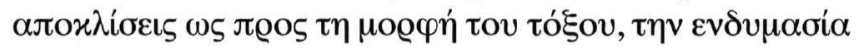

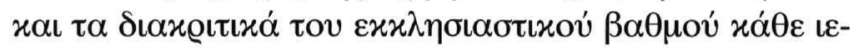

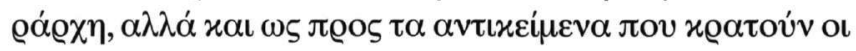

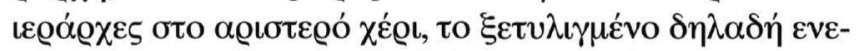

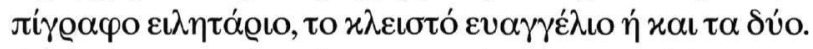

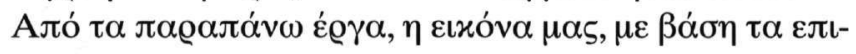

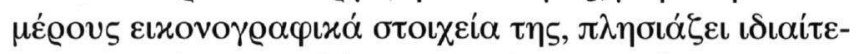

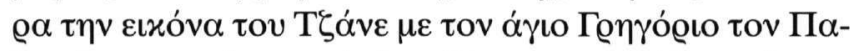

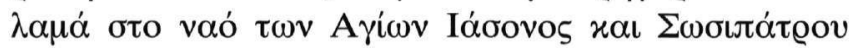

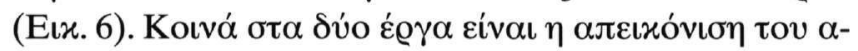

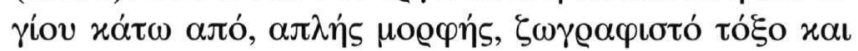

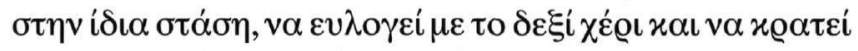

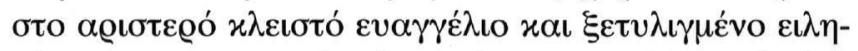

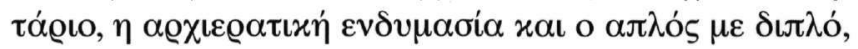

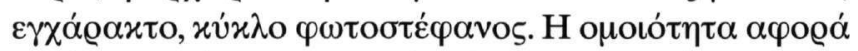

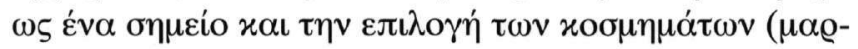

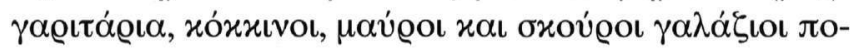

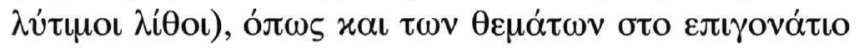

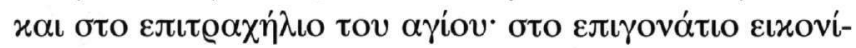

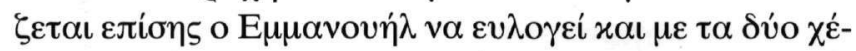

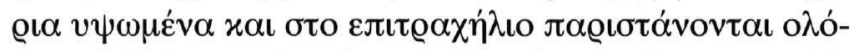

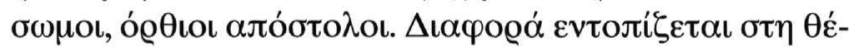

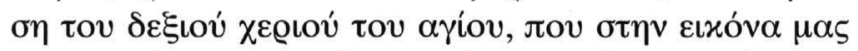

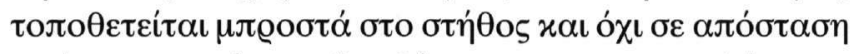

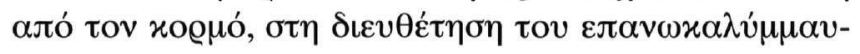

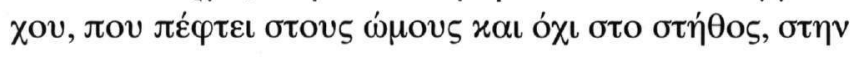

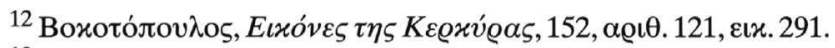

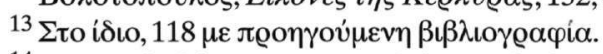

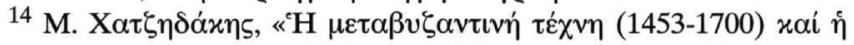

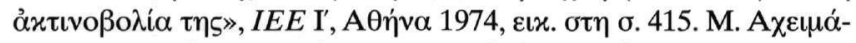

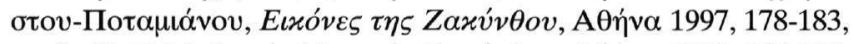

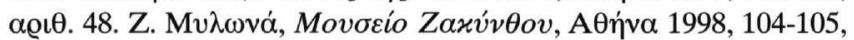

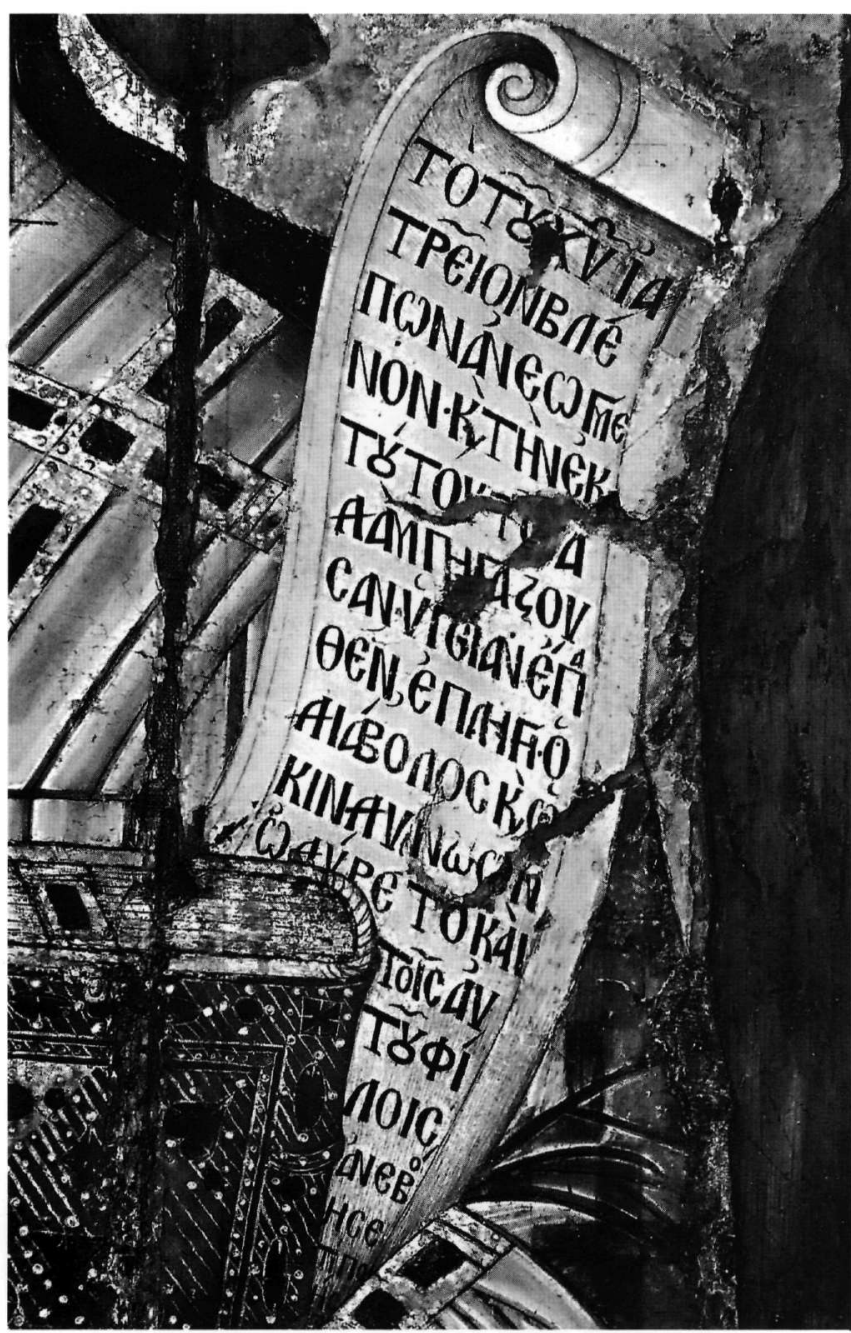

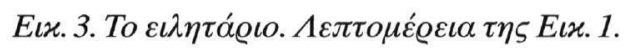

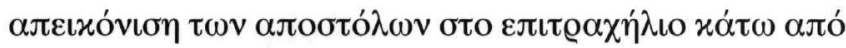

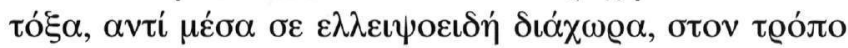

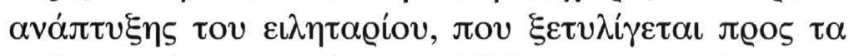

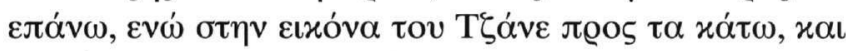

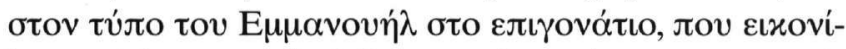

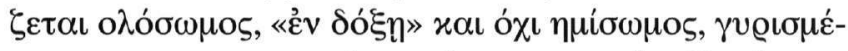

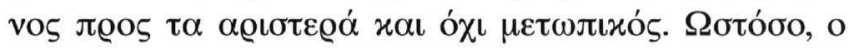

\footnotetext{
117-119, $\alpha \varrho \iota \theta .19$.

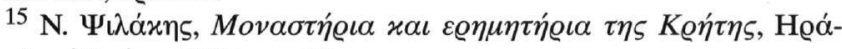

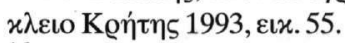

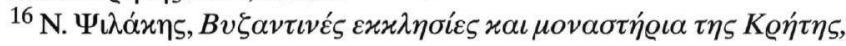

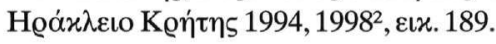




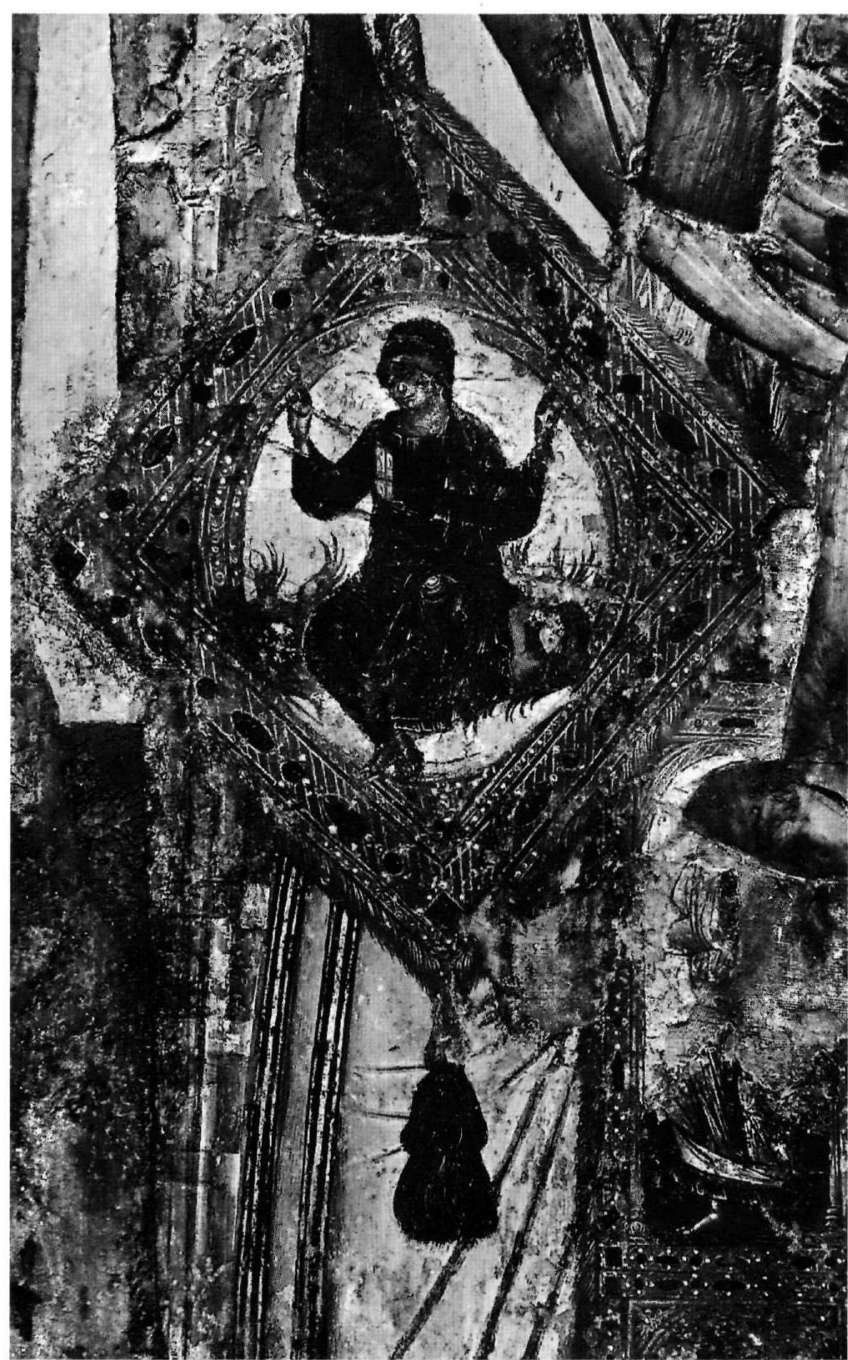

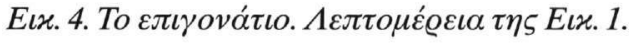

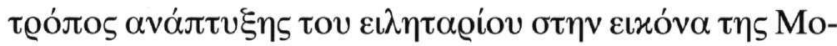

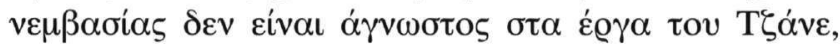

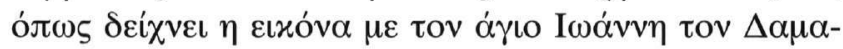

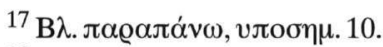

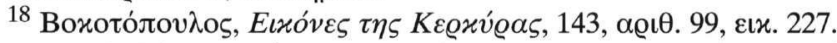

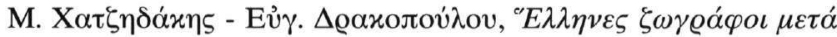

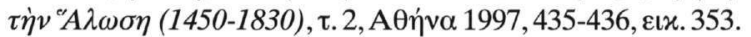

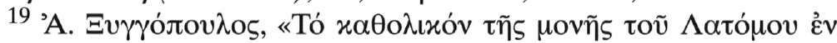

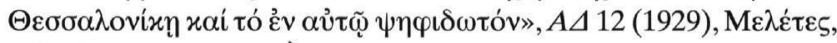
142-180. Ch. Diehl, "À propos de la mosaïque d'Hosios David à

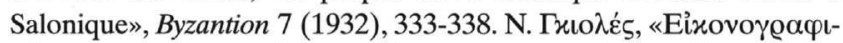

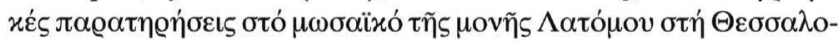

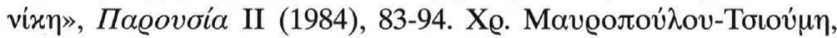

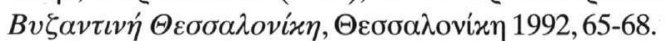

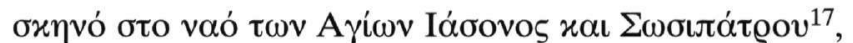

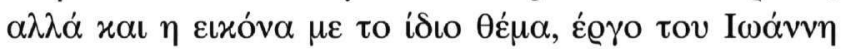

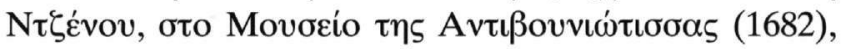

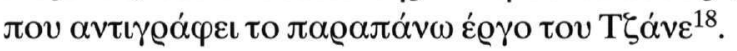

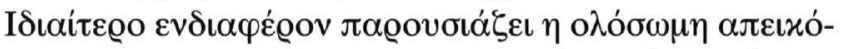

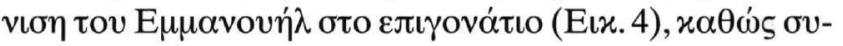

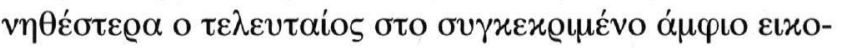

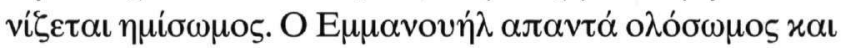

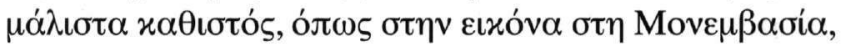

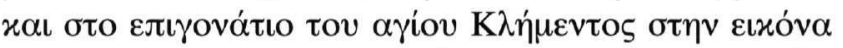

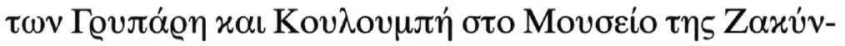

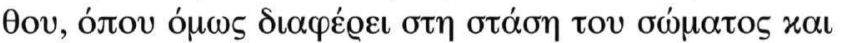

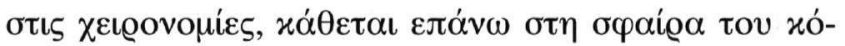

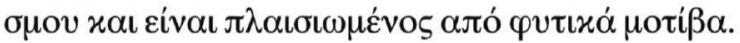

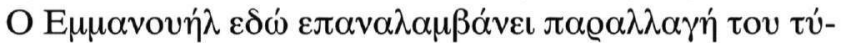

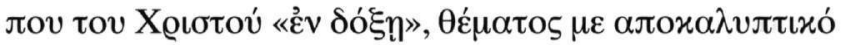

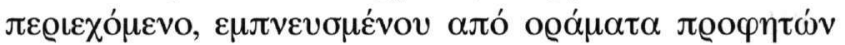

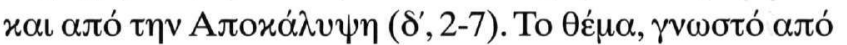

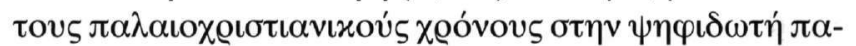

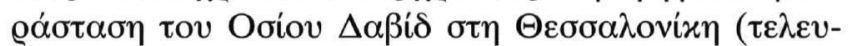

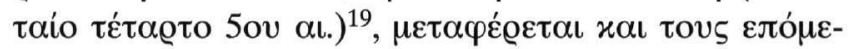

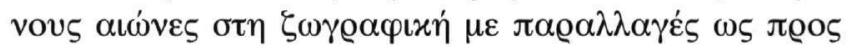

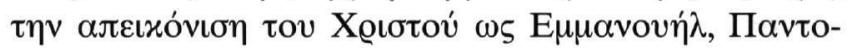

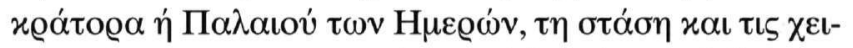

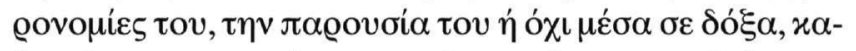

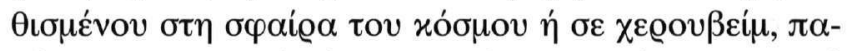

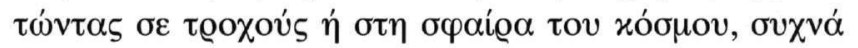

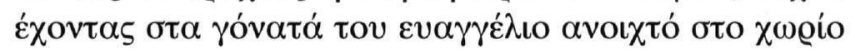

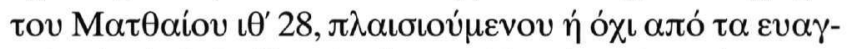

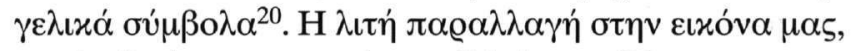

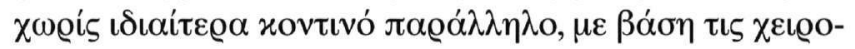

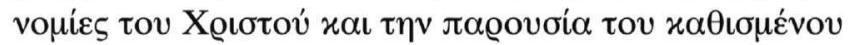

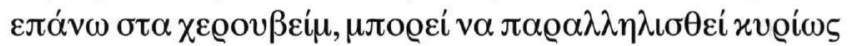

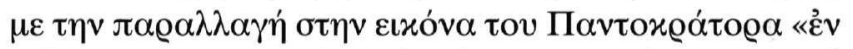

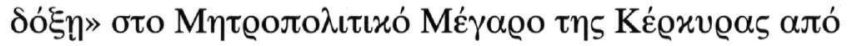

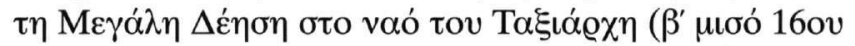

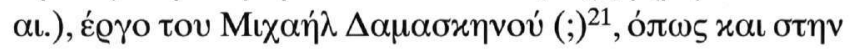

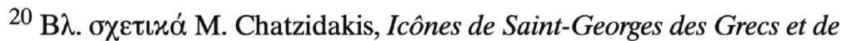

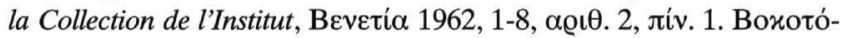

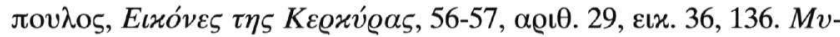

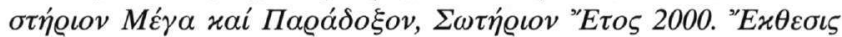

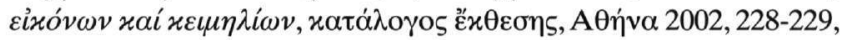

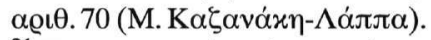

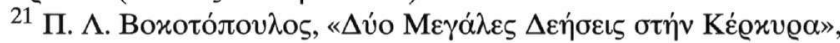

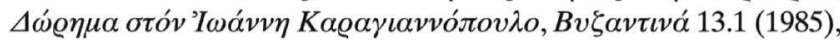

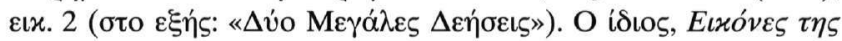

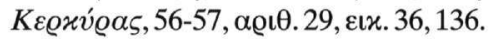




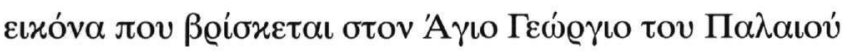

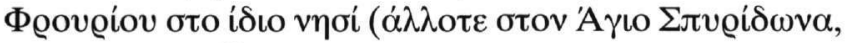
$\mu \varepsilon \dot{\sigma} \sigma \alpha 17 \mathrm{ov} \alpha \mathrm{u}.)^{22}$.

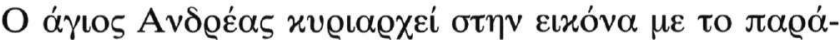

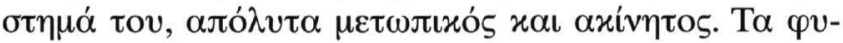

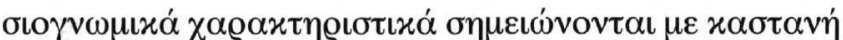

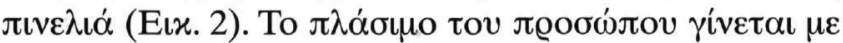

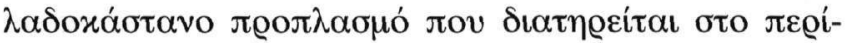

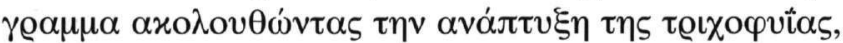

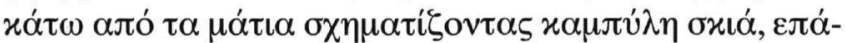

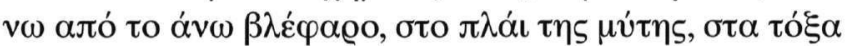

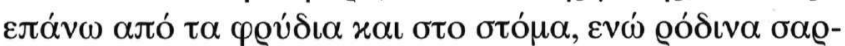

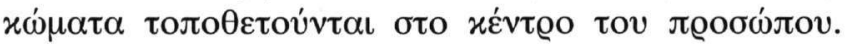

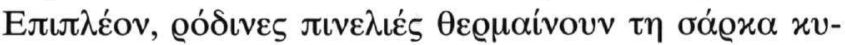

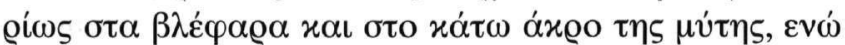

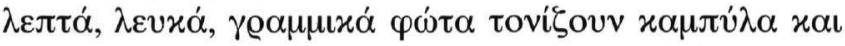

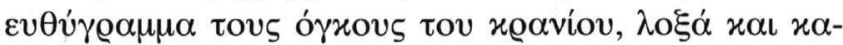

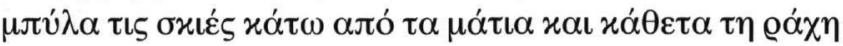

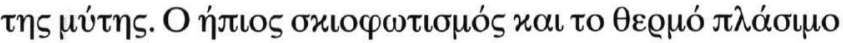

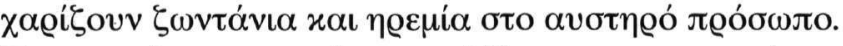

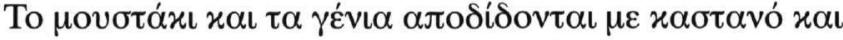

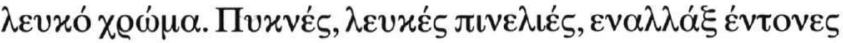

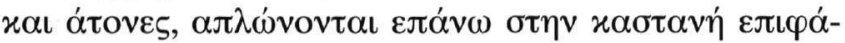

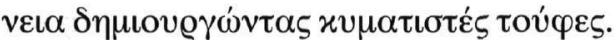

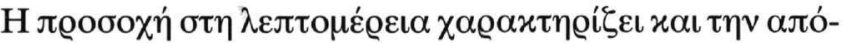

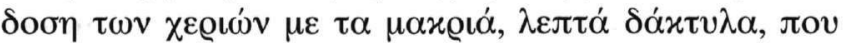

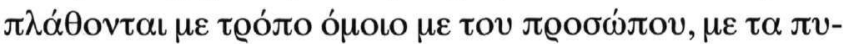

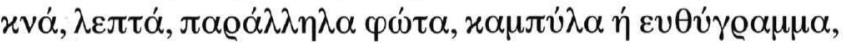

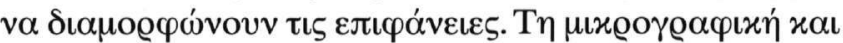

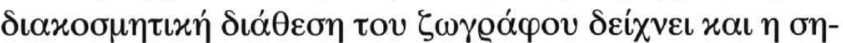

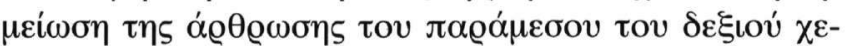

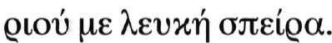

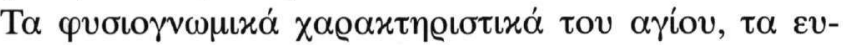

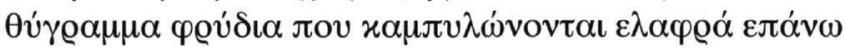

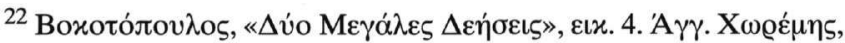

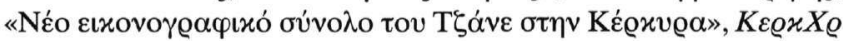

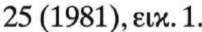

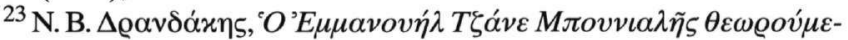

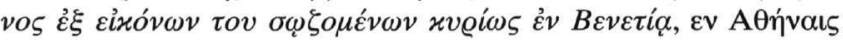
1962,17-24, $\alpha \varrho \imath \theta .1, \pi i v .1$.

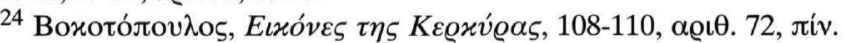
50.

${ }^{25} \Sigma$ тo íoı, 111-112, $\alpha \varrho \bullet \theta .75, \pi i v .53$.

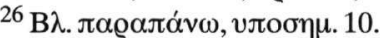

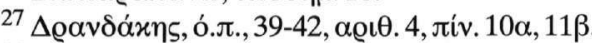

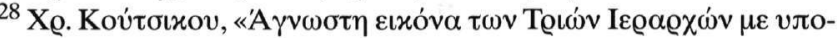

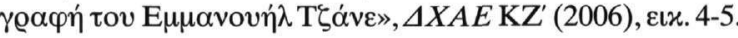

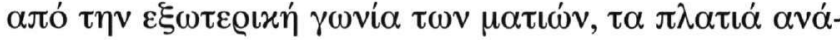

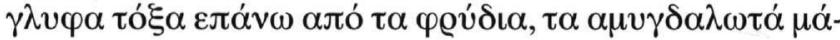

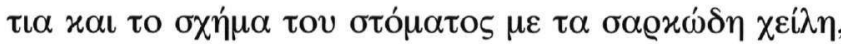

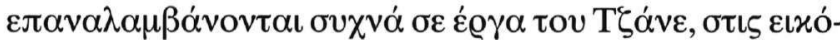

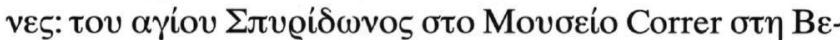

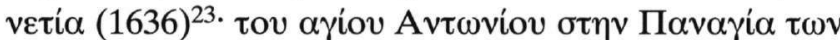

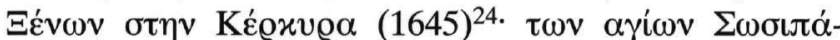

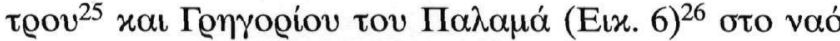

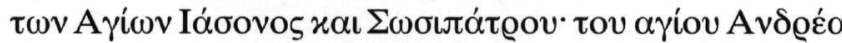

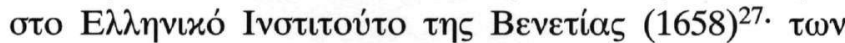

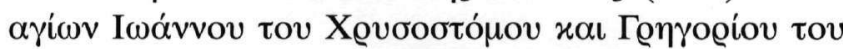

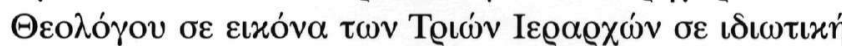

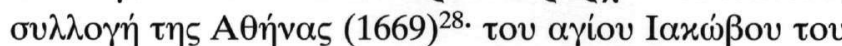

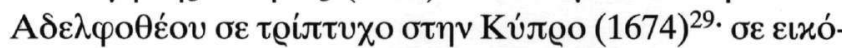

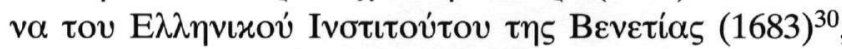

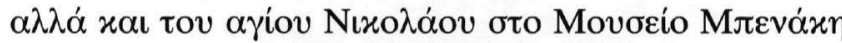

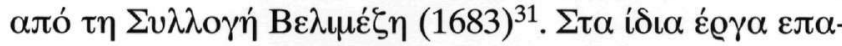

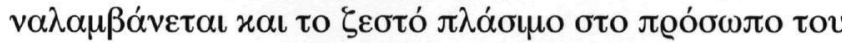

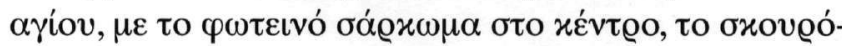

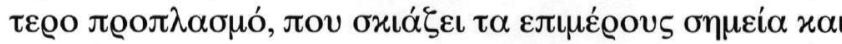

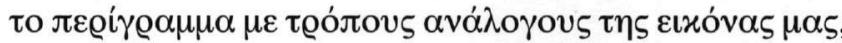

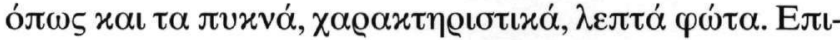

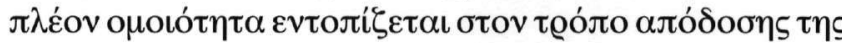

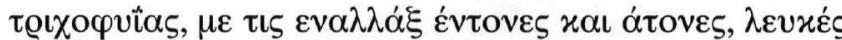

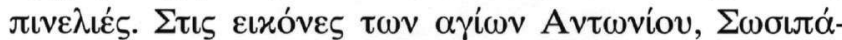

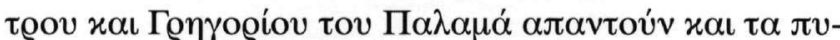

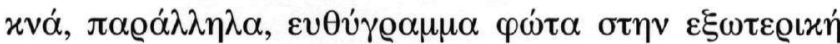

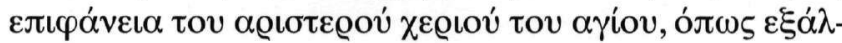

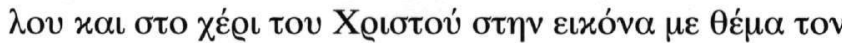

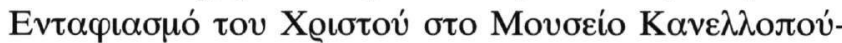

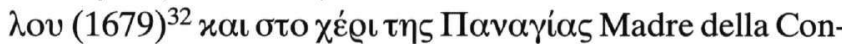

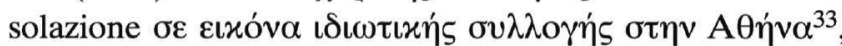

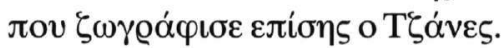

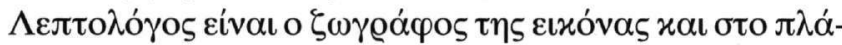

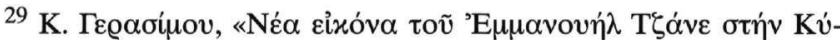
$\pi \varrho 0 », K v \pi \varrho \Sigma \pi \Xi \Sigma \mathrm{T}^{\prime}(2002), \varepsilon 1 x .3$

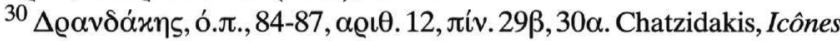

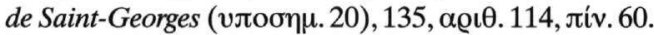

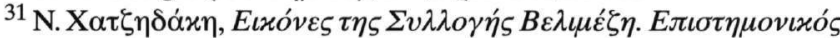

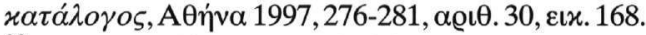

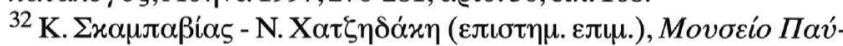

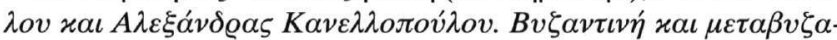

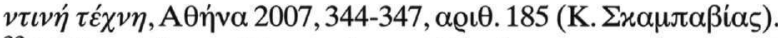

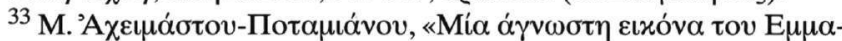

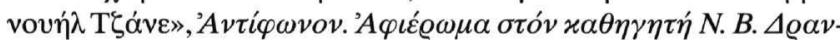

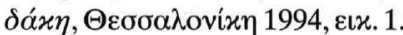




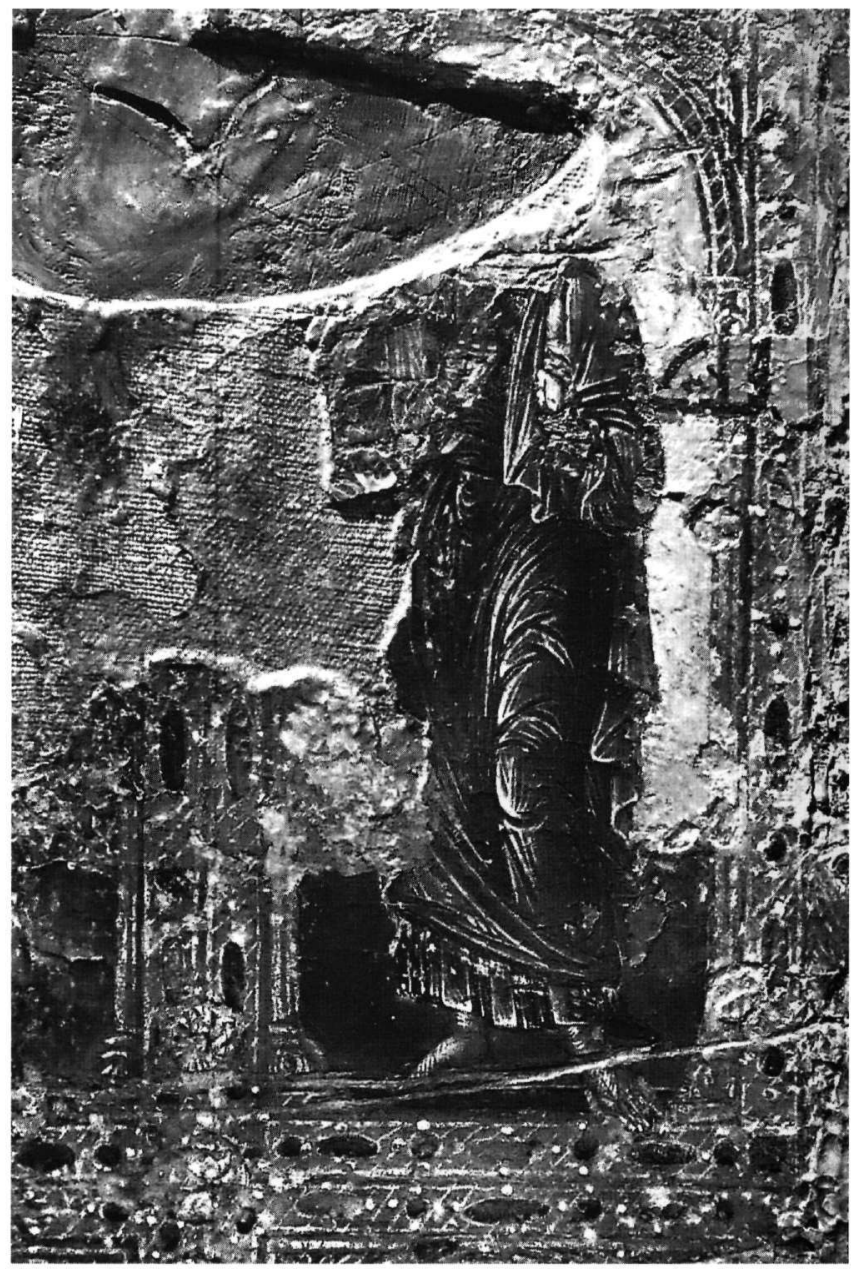

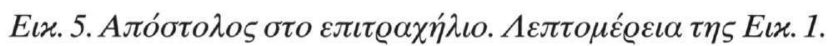

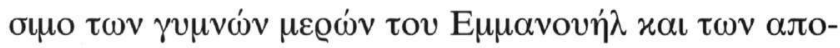

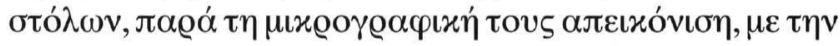

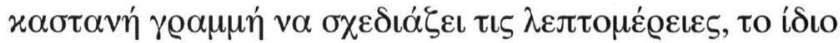

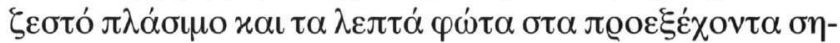

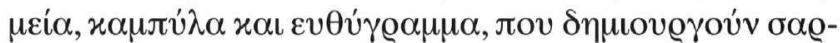

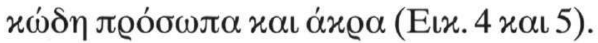

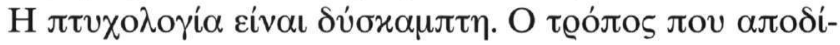

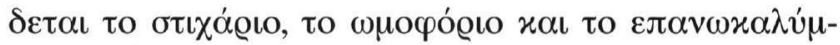

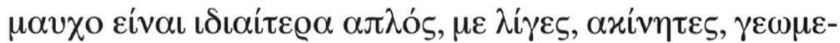

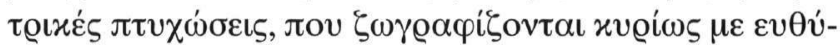

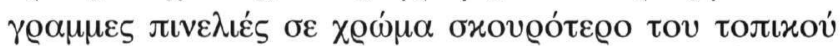

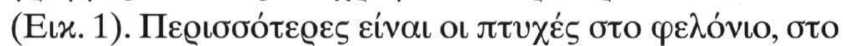

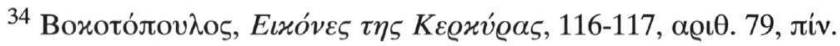
220.
}

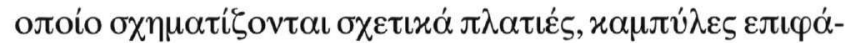

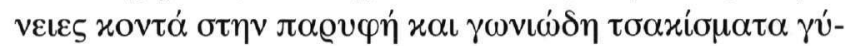

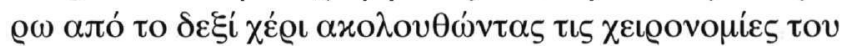

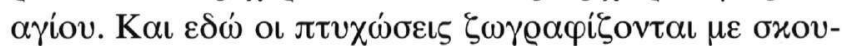

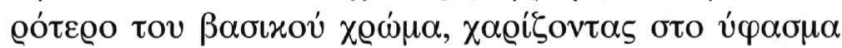

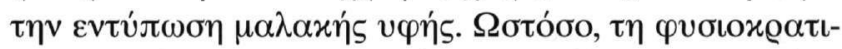

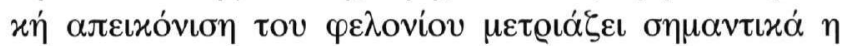

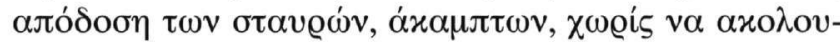

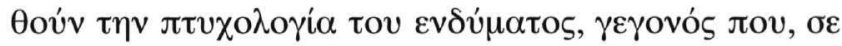

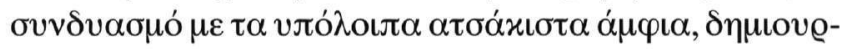

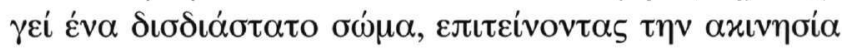

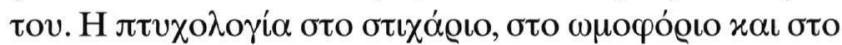

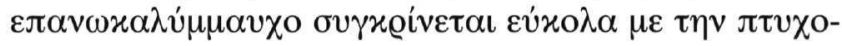

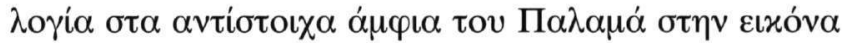

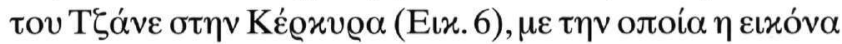

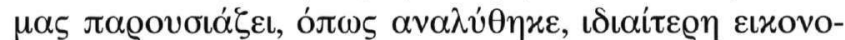

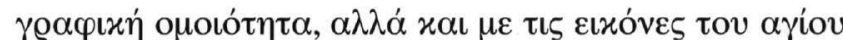

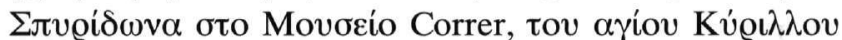

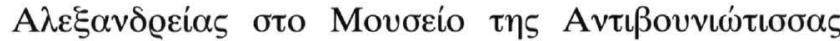

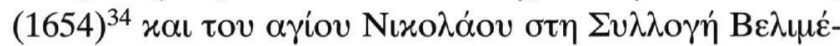

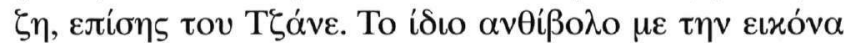

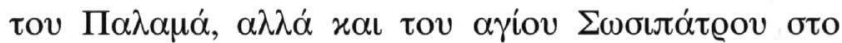

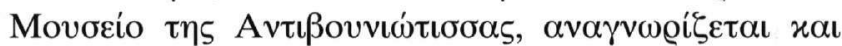

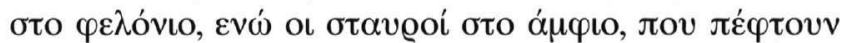

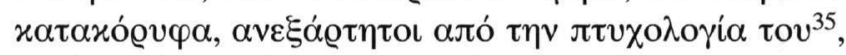

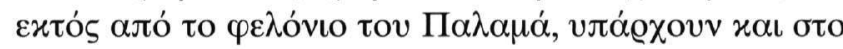

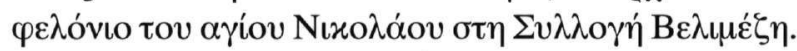

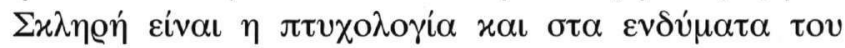

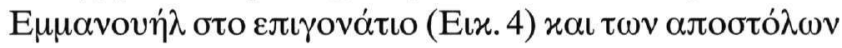

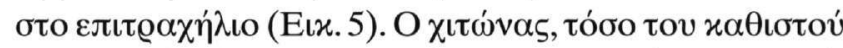

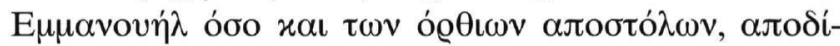

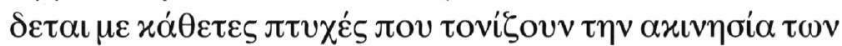

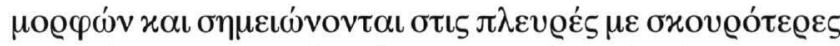

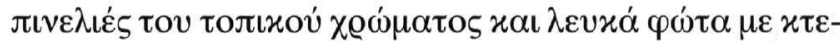

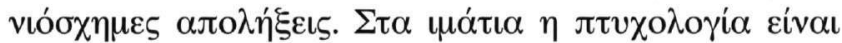

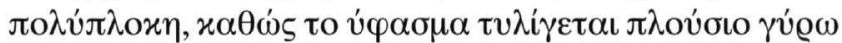

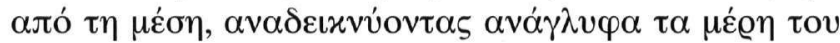

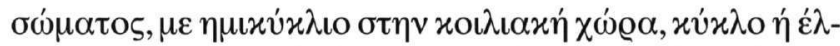

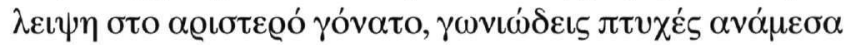

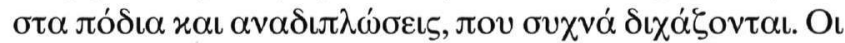

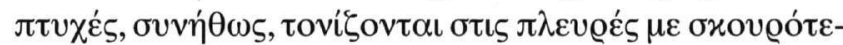

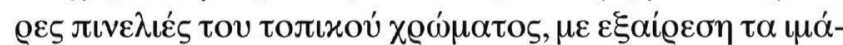

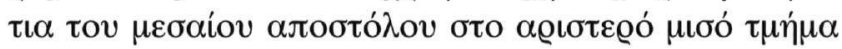

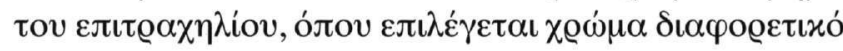

\footnotetext{
${ }^{35} \Sigma$ тo í́ıı, 119.
} 


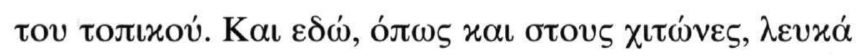

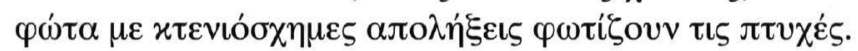

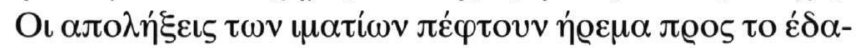

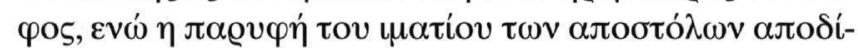

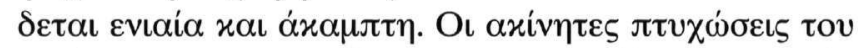

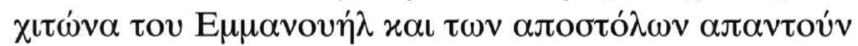

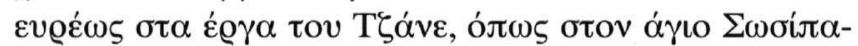

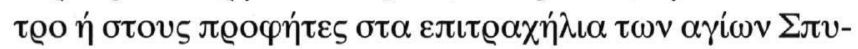

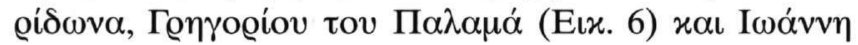

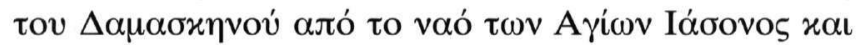

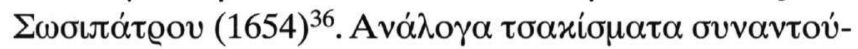

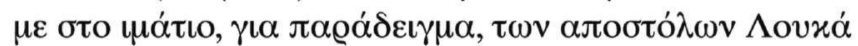

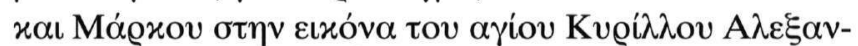

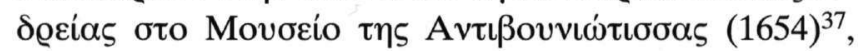

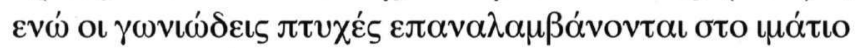

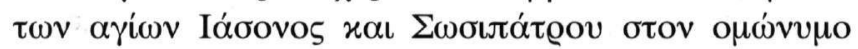

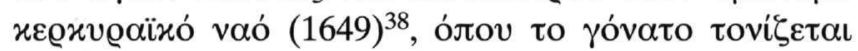

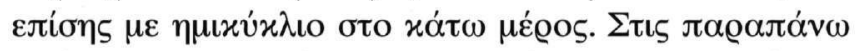

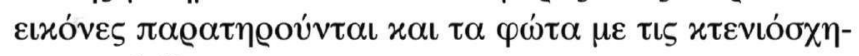
$\mu \varepsilon \varsigma \alpha \pi \mathrm{\alpha} \lambda \dot{\eta} \xi \varepsilon \iota \varsigma$.

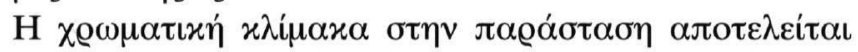

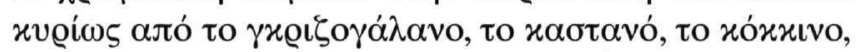

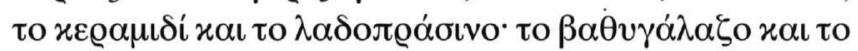

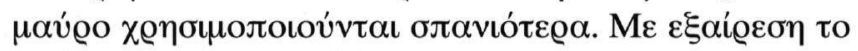

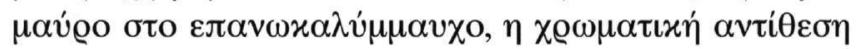

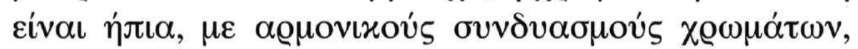

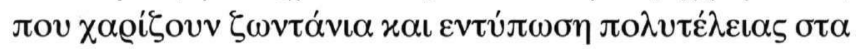

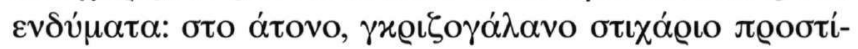

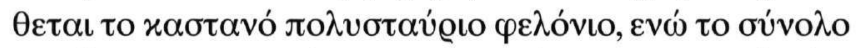

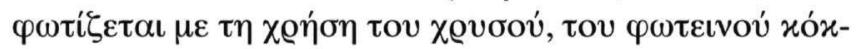

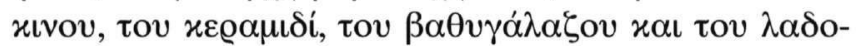

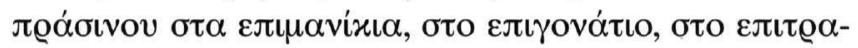

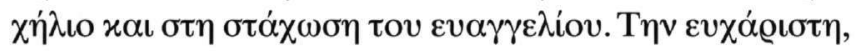

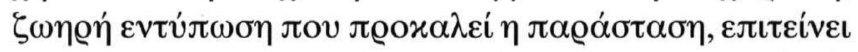

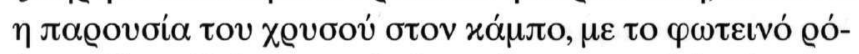

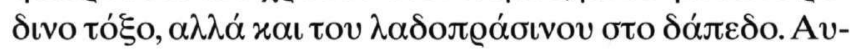

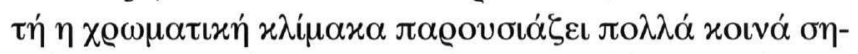

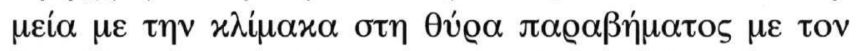

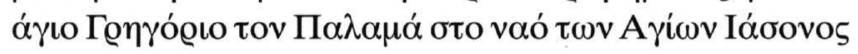

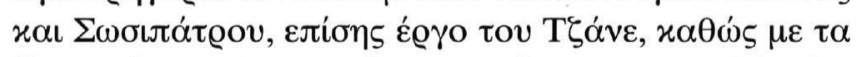

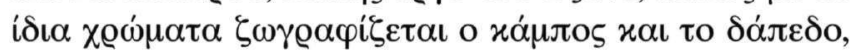

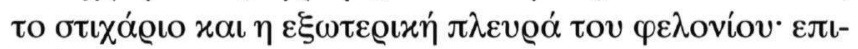

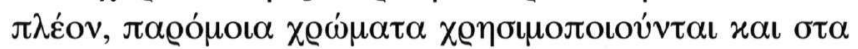

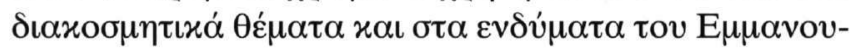

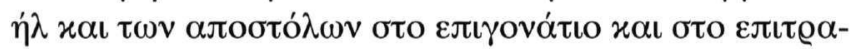

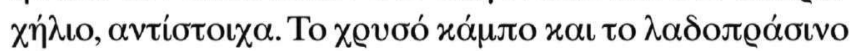

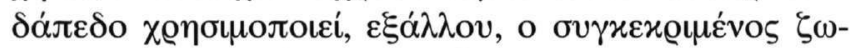

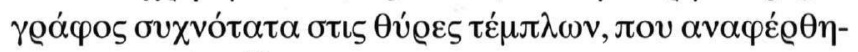

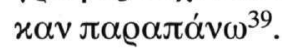

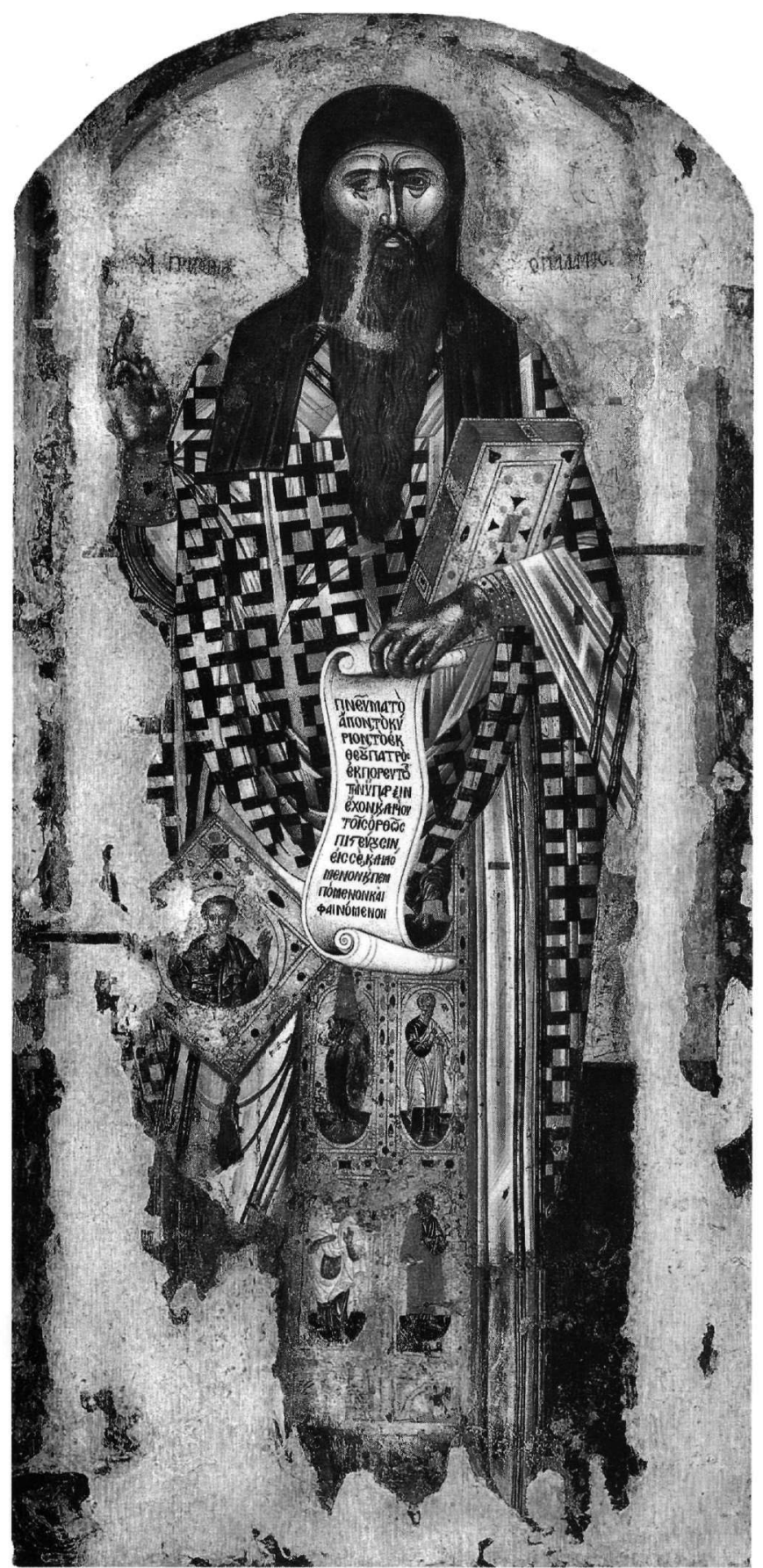

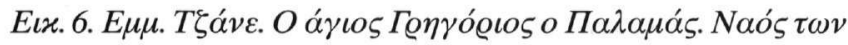

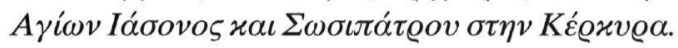

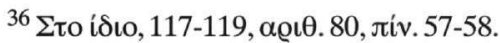

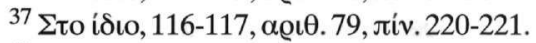

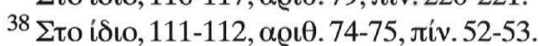

${ }^{39} \mathrm{~B} \lambda$. $\pi \alpha \varrho \alpha \pi \alpha ́ v \omega, 262-263$.
} 


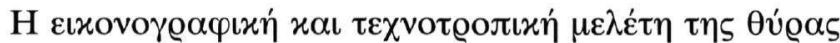

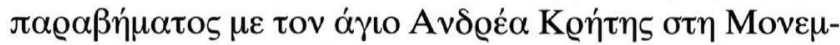

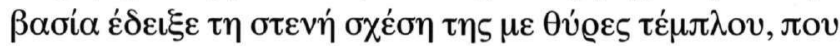

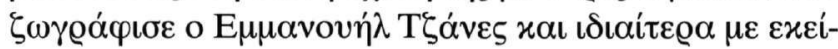

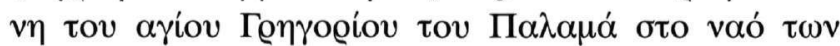

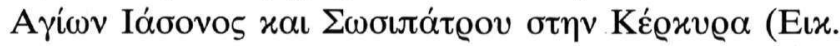

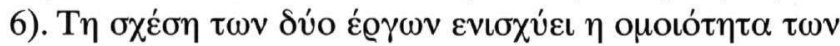

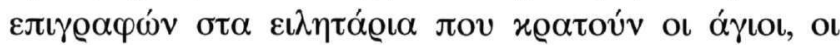

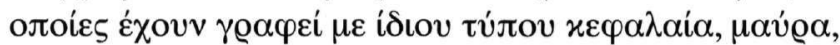

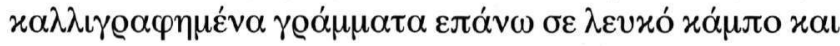

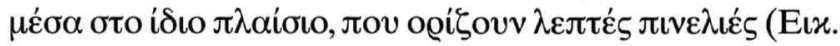

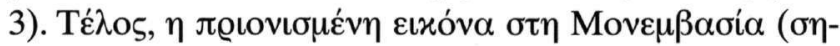

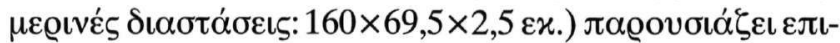

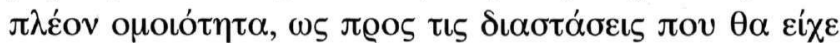

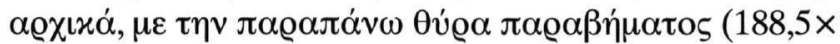

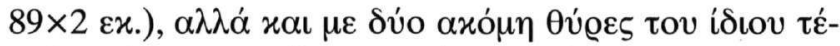

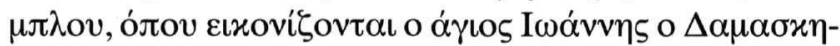

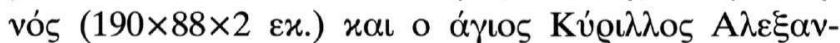

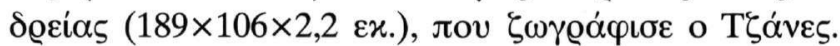

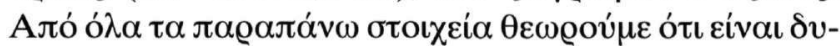

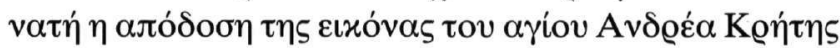

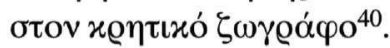

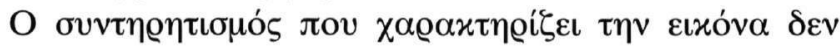

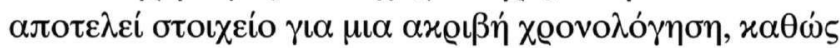

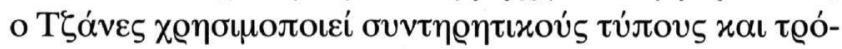

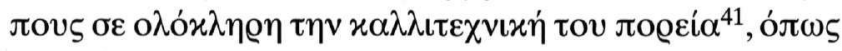

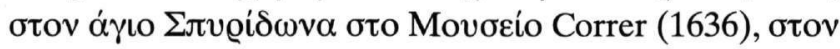
á

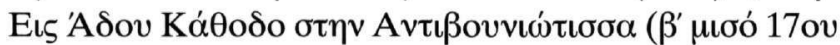

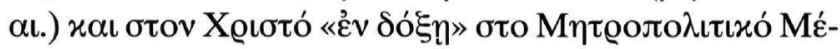

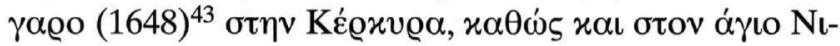

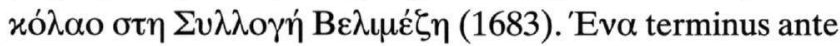

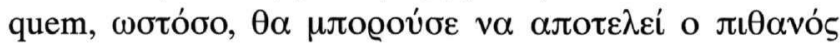

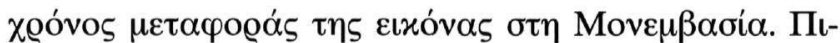

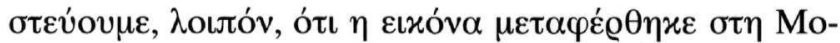

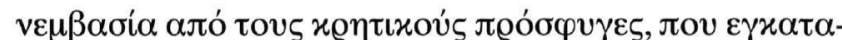

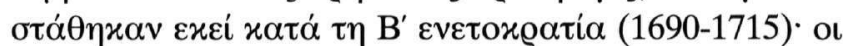

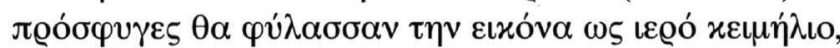

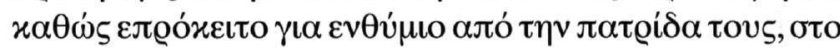

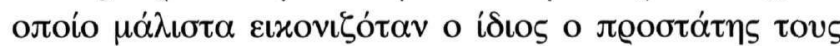

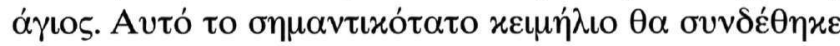

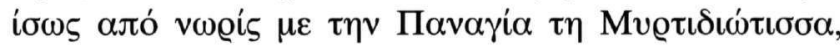

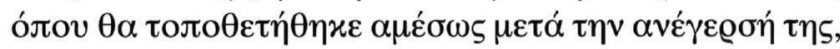

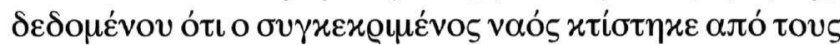

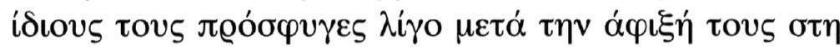

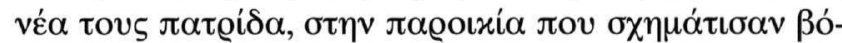

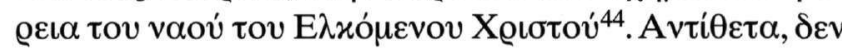

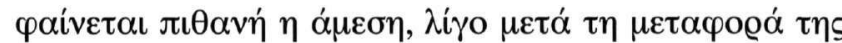

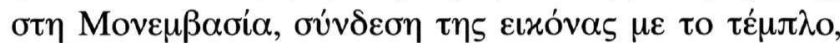

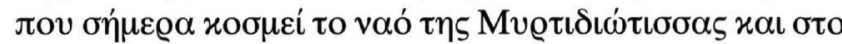

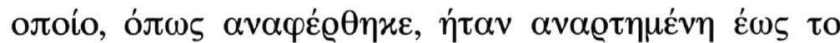

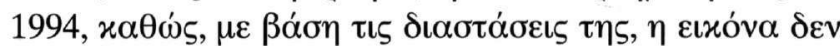

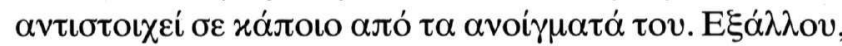

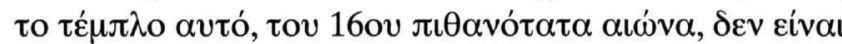

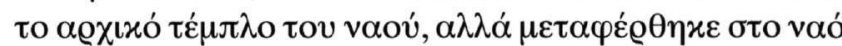

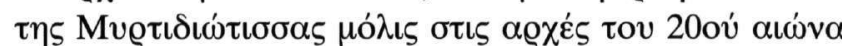

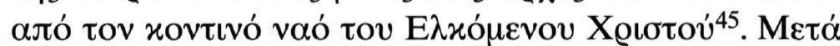

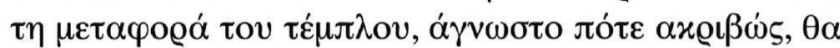

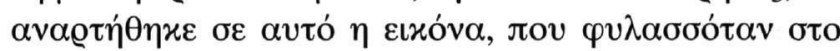

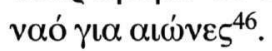

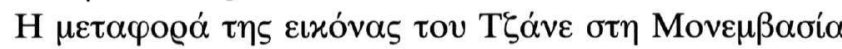

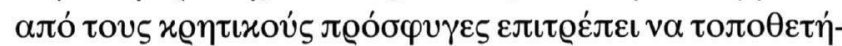

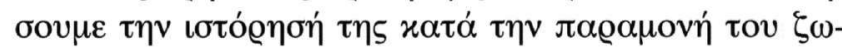

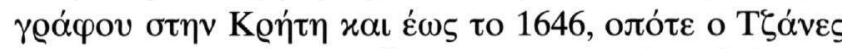

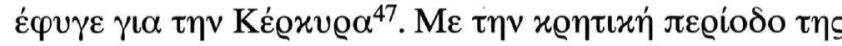

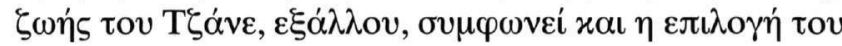

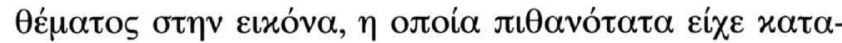

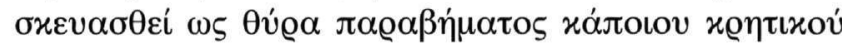
voov́.

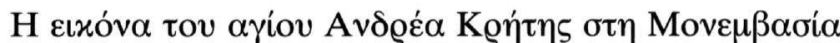

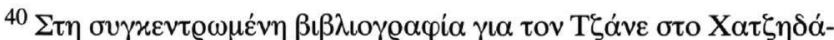

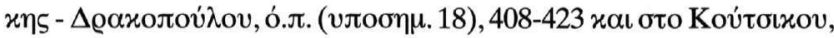

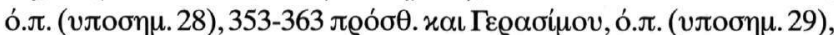

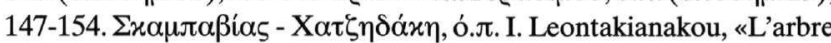
de Jessé avec la Vierge et ses parents : création et contenu dogmatique d'une icône d'Emmanuel Tzanès à l'Institut Hellénique de Venise»,

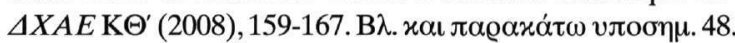

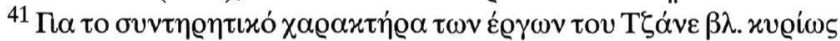

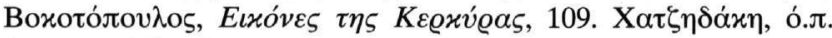
(vлоопи. 31), 278.

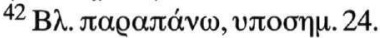

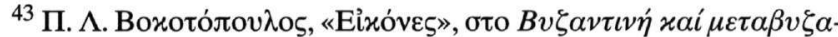

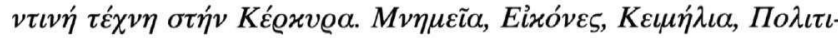

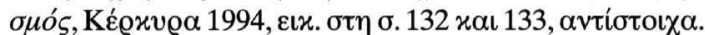

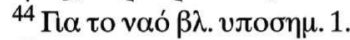

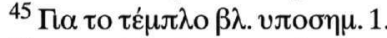

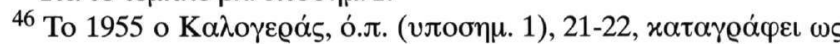

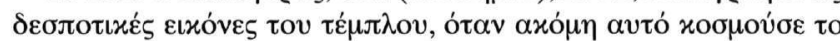

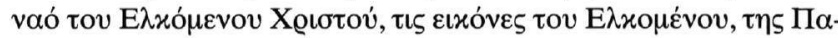

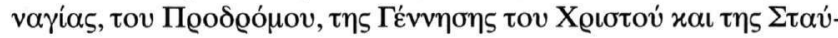

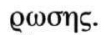

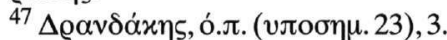




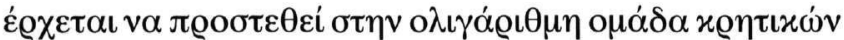

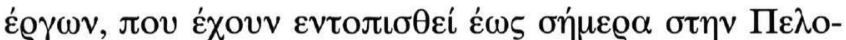

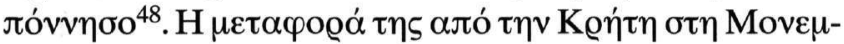

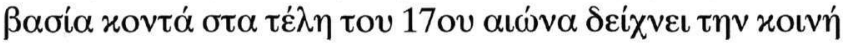

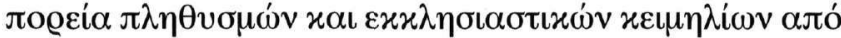

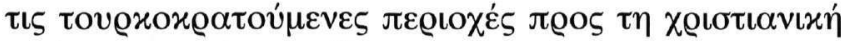

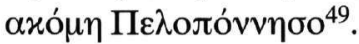

\section{Maria Agrevi}

\section{AN UNKNOWN ICON OF ST ANDREW OF CRETE AT MONEMVASIA, PAINTED BY EMMANUEL TZANES}

\begin{abstract}
$\mathrm{T}$ he icon of St Andrew of Crete (Fig. 1) comes from the church of the Panayia Myrtidiotissa at Monemvasia, where it hung on the iconostasis until 1994. It is now kept in the church of Christ Helkomenos. The icon now measures $160 \times 69.5 \times 2.5 \mathrm{~cm}$., but all four sides have been sawn off. Its large dimensions before parts of the wood were cut away, combined with the holes to attach a lock in about the middle of the left side, suggest that it was originally a door of one of the parabemata.

St Andrew, rendered life size, standing and frontally, wearing the vestments of a high priest, is projected beneath a painted arch supported on two slender columns. He blesses
\end{abstract}

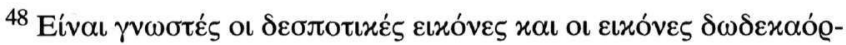

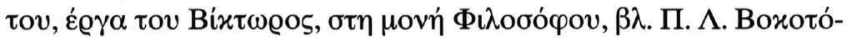

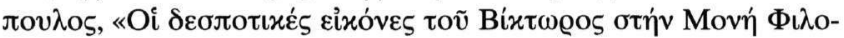

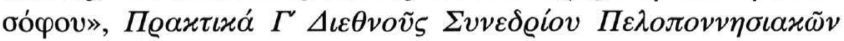

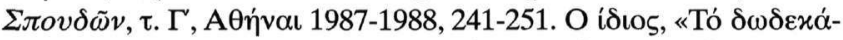

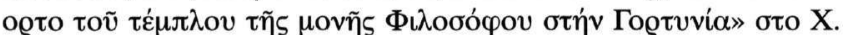

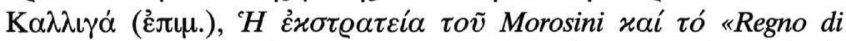

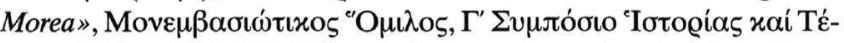

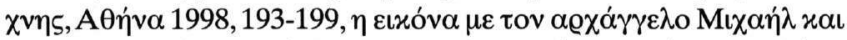

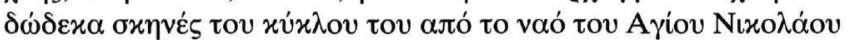

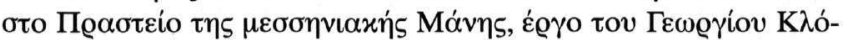

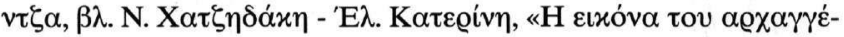

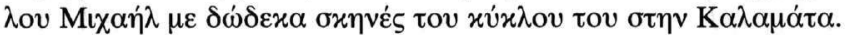

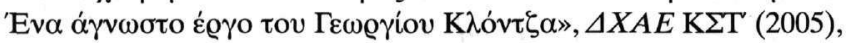

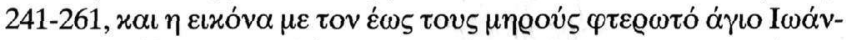

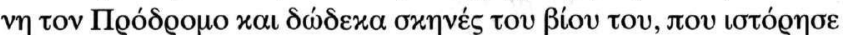

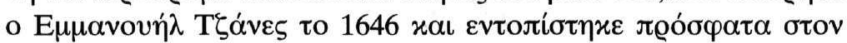

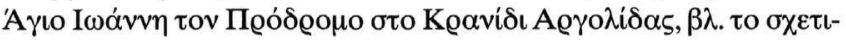

with his right hand and holds a closed lectionary in his left. A troparion of the Great Kanon composed by the saint himself is written on the scroll unfurled behind the lectionary. In iconographic terms, St Andrew repeats a type found in Post-Byzantine painting in large icons that served as templon doors; its closest parallel is the icon of St Gregory Palamas in the church of Sts Jason and Sosipatros on Corfu, which is the work of Emmanuel Tzanes. This icon, like other works by the same painter, repeats the stylistic elements of the representation: the facial features of the saint, the warm modelling of the face and exposed areas of flesh with dense, characteristically delicate highlights on projecting

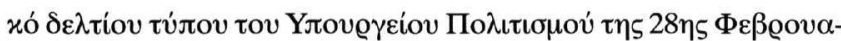

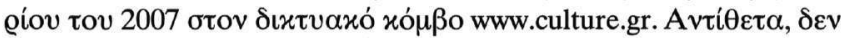

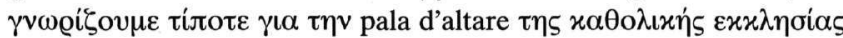

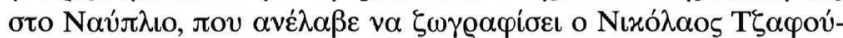

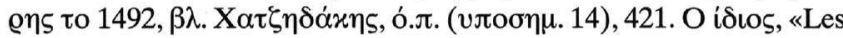
débuts de l'école crétoise et la question de l'école dite italogrecque»,

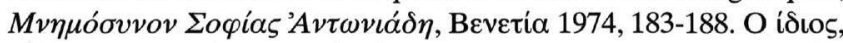

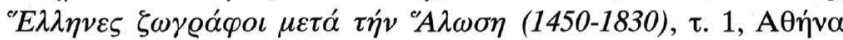
1987, 80, 292-294.

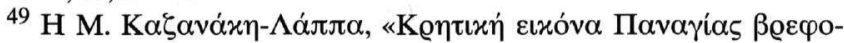

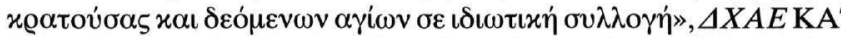

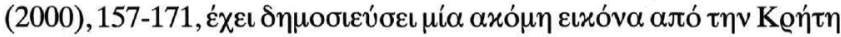

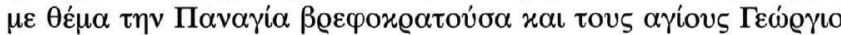

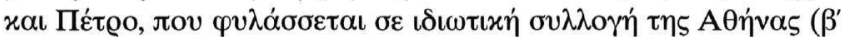

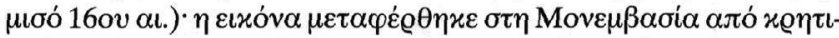

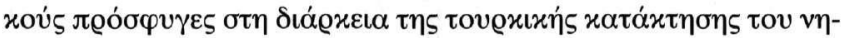

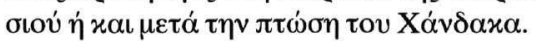


parts, the treatment of the hair with alternately thick and thin white brushstrokes, the geometric, rigid drapery that intensifies the body's lack of mobility, the annotation of the folds at the sides, usually with darker brushstrokes of the local colour lit by white lights with comb-like projections, the colour scale, and the painter's miniature, decorative intent.

The close relationship between the parabema door bearing the depiction of St Andrew of Crete and that with St Gregory Palamas in the church of Sts Jason and Sosipatros, by Emmanuel Tzanes (Fig. 6), enables the Monemvasia door to be assigned to the Cretan painter. The connection between the two works is strengthened by the similarity between the inscriptions on the scrolls held by the two saints, and also between the probable original dimensions of the sawn-down icon of Monemvasia (present dimensions $160 \times 69.5 \times 2.5$ $\mathrm{cm}$.) and those of the above parabema door $(188.5 \times 89 \times 2$ $\mathrm{cm}$.) and the other two doors from the same templon, depicting St John of Damascus $(190 \times 88 \times 2 \mathrm{~cm}$. $)$ and St Cyril of Alexandria $(189 \times 106 \times 2.2 \mathrm{~cm}$. $)$, also by Tzanes.

The icon of St Andrew of Crete was probably brought to Monemvasia by Cretan refugees who settled there during the second period of Venetian rule (1690-1715). The refugees will have kept the icon as a sacred heirloom, since it was a souvenir of their homeland, and one, indeed, that de- picted their patron saint himself. This important heirloom was possibly associated at an early date with the church of the Panayia Myrtidiotissa, in which it will have been placed immediately after its erection, since the church was built by the refugees themselves just after they arrived in their new home, in the neighbourhood in which they lived to the north of the church of Christ Helkomenos. It seems less likely, however, that the icon was placed on the templon that now adorns the church of the Myrtidiotissa (on which it hung until 1994) immediately after it was taken to Monemvasia, since it does not fit any of the openings of the templon. This templon, moreover, which probably dates from the 16th century, is not the original templon of the church, but was transferred to the Myrtidiotissa in the early 20th century from the nearby church of Christ Helkomenos. The present icon, which was kept in the church for centuries, will have been hung on the templon after it was moved to the church.

The fact that the icon by Tzanes was brought to Monemvasia by Cretan refugees enables us to assign the execution of it to the period when the painter lived on Crete, down to 1646, when he left for Corfu. The choice of subject for the icon, which was probably painted as a parabema door for a Cretan church, is also appropriate to the Cretan period of Tzanes' life. 